Pacific Journal of Mathematics

ON THE EXISTENCE OF CAPILLARY FREE SURFACES IN 


\title{
ON THE EXISTENCE OF CAPILLARY FREE SURFACES IN THE ABSENCE OF GRAVITY
}

\author{
JIN-TzU CheN
}

\begin{abstract}
If we were to put water into a glass cylindrical container of circular cross section and transport it to outer space, the surface of the water would be uniquely determined as a lower hemisphere. Concus and Finn [1] have shown that if the circular cross section is replaced by a square section then no such surface can exist as a graph of a function. The question then arises, for what kind of cross section can we expect the existence of a surface in the form of a graph?
\end{abstract}

Mathematically, this question can be formulated as follows: Let $\Omega$ be the cross section of the cylinder and $u$ be a capillary free surface defined over $\Omega$. By the least action principle of physics, $u$ would minimize the energy functional

$$
E[u]=\sigma \iint_{\Omega} \sqrt{1+|\nabla u|^{2}} d x d y-\sigma \lambda \int_{\Sigma} u d s
$$

subject to the volume constraint

$$
\iint_{\Omega} u d x d y=\text { constant }
$$

where $\Sigma$ is the boundary of $\Omega, d s$ is the arc length measure on $\Sigma$ and $\nabla u$ is the gradient of $u$. The physical interpretation of (1.1) is as follows: The first term gives the potential energy in the free surfaces; the constant $\sigma$ is referred to as the surface tension. The second term gives the wetting energy due to the boundary adhesion. The dimensionless constant $\lambda$ satisfies $|\lambda| \leqq 1$ and depends on the material of the wall and the fluid. For glass and water, $\lambda$ is close to 1 , and for glass and mercury, $\lambda$ is negative. We mention that the case $\lambda>1$ corresponds physically to a situation in which adhesion dominate, so that the fluid would spread out along the walls and no equilibrium surface would exist. This phenomenon is observed, e.g., with liquid helium and glass.

The equilibrium condition $\delta E[u]=0$ under the constraint (1.2) is expressed by the Euler equations:

$$
\begin{gathered}
\operatorname{div} T u=H \text { in } \quad \Omega \\
T u \cdot \nu=\lambda \text { on } \Sigma
\end{gathered}
$$

where $T u\left\langle u_{x} / \sqrt{1+|\nabla u|^{2}}, u_{y} / \sqrt{1+|\nabla u|^{2}}\right\rangle, H$ is a constant which is 
twice the mean curvature of the capillary free surface, and $\nu$ is the unit outer normal of $\Sigma$.

If we introduce the angle $\gamma$ by letting $\gamma=\cos \gamma$, then (1.3) and (1.4) imply that $u$ is a surface of constant mean curvature which makes constant contact angle $\gamma$ with the wall; the angle $\gamma$ is measured inside the fluid, as shown in Figure 1.

It should be noted here that the constant $H$ connot be prescribed; it is implicity determined by the geometry of $\Omega$ and the contact angle $\gamma$, as follows by applying the divergence theorem to (1.3) and using (1.4):

$$
H=\frac{\Sigma}{\Omega} \cos \gamma .
$$

We use here the symbols $\Omega$ and $\Sigma$ to denote both a set and its measure. We may assume $0 \leqq \gamma \leqq \pi / 2$; for further physical and geometrical background information, see [8].

The following necessary condition for the existence of solution of (1.3) and (1.4) was first observed by Concus and Finn [3]:

Let $\Omega^{*}$ be any subdomain in $\Omega$ bounded by a simple curve $\Gamma$ and a subboundary $\Sigma^{*}$ of $\Sigma$, as shown in Figure 2. Integrate (1.3) over $\Omega^{*}$, using (1.4) and the divergence theorem we have

$$
\begin{aligned}
H \Omega^{*} & =\iint_{\Omega^{*}} \operatorname{div} T u d x d y \\
& =\int_{\Sigma^{*+\Gamma}} T u \cdot \nu d s \\
& =\int_{\Sigma^{*}} T u \cdot \nu+\int_{\Gamma} T u \cdot \nu d s \\
& =\Sigma^{*} \cos \Gamma+\int_{\Gamma} T u \cdot \nu d s
\end{aligned}
$$

substituing (1.5) and using the fact $|T u| \leqq 1$, we obtain:

$$
-\Gamma \leqq \frac{\Omega^{*}}{\Omega} \Sigma \cos \Gamma-\Sigma^{*} \cos \Gamma \leqq \Gamma
$$

or equivalently

$$
\left|\frac{\Sigma^{*}}{\Sigma}-\frac{\Omega^{*}}{\Omega}\right| \cos \gamma \leqq \frac{\Gamma}{\Sigma}
$$

for any $\Gamma$.

For a solution of (1.3) and (1.4) to exist, it is necessary that (1.5) hold for every choice of $\Gamma$. As was shown in [3], an arbitrary $\Sigma$ can be changed by an arbitrary small deformation into a boundary for which (1.6) will fail for some $\Gamma$. 
Condition (1.6) was later shown by Giusti [13] to be not only necessary, but (almost) sufficient. For most purpose, Giusti required

$$
\left|\frac{\Sigma^{*}}{\Sigma}-\frac{\Omega^{*}}{\Omega}\right| \cos \gamma<(1-\varepsilon) \frac{\Gamma}{\Sigma}
$$

for some $\varepsilon>0$ and any $\Gamma$.

Obviously (1.7) is implied by

$$
\left|\frac{\Sigma}{\Sigma}-\frac{\Omega}{\Omega}\right| \leqq \frac{\Gamma}{\Sigma}
$$

for every contact angle $0<\gamma \leqq \pi / 2$. Thus if we could characterize those domains for which (1.8) holds, then (1.3) and (1.4) will have solution on them for any prescribed contact angle $\gamma>0$.

The difficulty of using (1.7) and (1.8) is that one must verify them for every possible $\Gamma$. It is shown in [15] that if $\Omega$ is convex, then (1.8) follows from the following curvature condition on $\Sigma$ :

$$
K(p) \leqq \frac{\Sigma}{\Omega}
$$

for every $p \in \Sigma$, where $K(p)$ denotes the curvature of $\Sigma$ at $p$. For nonconvex domains there appears to be no analogous criterion, c.f. the examples in Finn and Giusti [11].

Instead of thinking about the curvature conditions on $\Sigma$, Finn [9] gave the following vector field condition on $\Omega$ which is equivalent to (1.7):

THEOREM (Finn). The condition (1.7) holds for arbitrary $\Gamma \subset \Omega$ whenever there is a vector field $W(x, y)$ in $\Omega$ satisfying

$$
\begin{gathered}
\operatorname{div} W=\frac{\Sigma}{\Omega} \text { in } \Omega \\
w \cdot \nu=1 \text { on } \Sigma \\
\operatorname{Sup}_{\Omega}|w|<\frac{1}{\cos \gamma} .
\end{gathered}
$$

By using this theorem, Finn proved the existence of solution over triangles, tetrahedrons, parallelograms and some special polygons by constructing the vector field directly. He required $\alpha+\gamma \geqq \pi / 2$, where $2 \alpha$ denotes the smallest angle of the vertices of the domain (In case $\alpha+\gamma<\pi / 2$, Concus and Finn [1] have proved that no solutions exist.) For general domains a difficult point in connection with the use of Finn's theorem.

In this paper, we shall use variational method to reduce the class 
of curves on which we need to test Concus-Finn-Giusti's condition (1.7) to some special curves $\Gamma$, which have property that if (1.7) holds for these special curves $\Gamma$, then it holds for all possible curves. This enables us to state following existence theorem depending on the geometric properties of $\Omega$ :

THEOREM 4.1. If a circle of radius $R=\Omega / \Sigma$ can be rotated along $\Sigma$ in the interior of $\Omega$ such that no antipodes of the circle lie on $\Sigma$, then (1.8) holds and hence a solution of (1.3), (1.4) exists in $\Omega$ for all $\gamma>0$.

Actually, we prove that (1.8) holds strictly for every $\Omega^{*} \neq \varnothing, \Omega$; by a theorem of Giusti (Theorem 1, [15]), this implies that a solution exists for $\gamma=0$.

The sufficient condition of Theorem 4.1 is also necessary for existence of the solution for domains without a "neck" of radius $R=\Omega / \Sigma$. (Here what we mean by a neck domain of radius $R$ is a domain which has two large components with narrow connection through which one cannot pass through a circle of radius $R$.) If such a "neck" appears, the necessity can fail. For example, a domain which is the union of two disks of equal radius will fail to satisfy the condition of Theorem 4.1 if the aperture is sufficiently small; however, as we shall show in $\S 5$, the solution nevertheless exists.

2. Isoperimetric inequalities. The purpose of this section is to provide some basic isoperimetric inequalities which will be used to prove theorems in the later sections.

A well known form of the classical isoperimetric inequality can be stated as follows:

Among all plane curves of prescribed arc length which pass through the two given $P, Q$; the circular arc bounds the maximum area between it and the line segment through $P$ and $Q$.

This result still holds if the line segment is replaced by a plane curve which does not have intersections with the circular arc of prescribed arc length.

The following lemma is a generalization of the classical isoperimetric inequality in a bounded domain.

LEMMA 2.1. Let $\Omega$ be a plane domain with piecewise smooth boundary $\Sigma$, let $P$ and $Q$ be two points on $\Sigma$ which divide $\Sigma$ into two parts $\Sigma_{1}, \Sigma_{2}$ and let $\Gamma$ be a curve in $\Omega$ joining $P$ and $Q$ with prescribed arc length less then $\Sigma_{1}$ and bounds with $\Sigma_{2}$ an area $\Delta(\Gamma)$. Then $\Delta(\Gamma)$ achieves its maximum in one of the following cases; 
(i) $\Gamma$ is a circular arc if one can draw a circular arc in $\Omega$ with the prescribed arc length

(ii) $\Gamma$ is a curve which coincides with some parts of $\Sigma$ and circular arcs of equal radii. Furthermore, if the end points of the circular arcs are smooth points of $\Sigma$, then the circular arcs are tangent to $\Sigma$ there except possibly for the initial points $P$ and $Q$. (Figure $3 \mathrm{a}, \mathrm{b}, \mathrm{c}$.

Proof. (Uniqueness). If one can draw a circular arc of the prescribed arc length in $\Omega, \Delta(\Gamma)$ achieve its maximum by the classical isoperimetric inequality. Suppose that is not the case. Since fixing any two points of $\Gamma$ and deforming the subarc of $\Gamma$ between these two points into a circular arc always increases the enclosed area, $\Gamma$ will consist of some pieces of $\Sigma$ and some circular arc in $\Omega$. Let $C_{i}, C_{j}$ be two circular arc with radii $r_{i}, r_{j}$ respectively. If $r_{i} \neq r_{j}$ we can cut $C_{i}$ and $C_{j}$ a little bit by two chords $l_{i}$ and $l_{j}$ respectively with $l_{i}=l_{j}$ (Figure 4). Let $S_{i}$ and $S_{j}$ be the small regions cut out from $C_{i}$ and $C_{j}$ respectively. Moving $S_{i}$ to $l_{j}$ and $S_{j}$ to $l_{i}$, one obtains a new curve $\Gamma^{\prime}$ which bounds a region with the same area as $\Gamma$, but now the subarcs of $\Gamma^{\prime}$ are not circular. Hence we can deform it into a circular arc and increase the area bounded. This proves that $r_{i}=r_{j}$.

Next, we want to prove that any circular $C_{i}$ is tangent to $\Sigma$ if the end points of $C_{i}$ are smooth points of $\Sigma$. Let $E$ be an end point of $C_{i}$ which is a smooth point of $\Sigma$ and an interior point of $\Gamma$. Let $A$ and $B$ be two points on $\Gamma$ in a neighborhood of $E$ such that $A \in \Sigma$, $B \in C_{i}$, and let $A$ be the origin of the coordinate system with $A B$ as $x$-axis (Figure 5). By taking a small neighborhood we can assume that $\Sigma$ is expressed as $y=g(x)$ in a neighborhood $\Sigma_{A F}$. Now let $l$ be the arc length of $\Gamma_{A B}$. We will use a variational method to show that $\Gamma_{A B}$ should be tangent to $\Sigma$ at $E$.

Let $(b, 0)$ and $\left(x_{0}, g\left(x_{0}\right)\right)$ be the coordinates of $B$ and $E$ respectively, and $y=f(x)$ be the equation of $\Gamma_{E B}$ with respect to this coordinate system, then

$$
\begin{aligned}
l & =\int_{0}^{x_{0}} \sqrt{1+g^{\prime 2}}(x) d x+\int_{x_{0}}^{b} \sqrt{1+f^{\prime 2}}(x) d x \\
\Delta & =\text { area bounded by } \Gamma_{A B} \text { and the } x \text {-axis } \\
& =\int_{0}^{x_{0}} g(x) d x+\int_{x_{0}}^{b} f(x) d x .
\end{aligned}
$$

We are going to maximize (2.2) under the constraint (2.1).

Let $\eta$ be a continuous differentiable function defined on $[0, b]$ with $\eta(b)=0$, and let 


$$
\begin{aligned}
I(\varepsilon)= & \int_{0}^{x_{0}}[g(x)+\varepsilon \eta(x)] d x+\int_{x_{0}}^{b}[f(x)+\varepsilon \eta(x)] d x \\
& +\lambda \int_{0}^{x_{0}} \sqrt{1+\left[g^{\prime}(x)+\varepsilon \eta^{\prime}(x)\right]^{2}} d x+\lambda \int_{x_{0}}^{b} \sqrt{1+\left[f^{\prime}(x)+\varepsilon \eta^{\prime}(x)\right]^{2}} d x
\end{aligned}
$$

then

$$
\begin{aligned}
\left.\frac{d I}{d \varepsilon}\right|_{c=0}= & \int_{0}^{x_{0}} \eta(x) d x+\int_{x_{0}}^{b} \eta(x) d x+\lambda \int_{0}^{x_{0}} \frac{g^{\prime}(x) \eta^{\prime}(x)}{\sqrt{1+g^{\prime 2}(x)}} d x \\
& +\lambda \int_{x_{0}}^{b} \frac{f^{\prime}(x) \eta^{\prime}(x)}{\sqrt{1+f^{\prime}(x)^{2}}} d x \\
= & \int_{0}^{b} \eta(x) d x-\lambda \int_{0}^{x_{0}} \frac{d}{d x}\left(\frac{g^{\prime}(x)}{\sqrt{1+g^{\prime 2}(x)}}\right) \eta(x) d x \\
& -\lambda \int_{x_{0}}^{b} \frac{d}{d x}\left(\frac{f^{\prime}(x)}{\sqrt{1+f^{\prime 2}(x)}}\right) \eta(x) d x \\
& +\left.\lambda \eta(x) \frac{g^{\prime}(x)}{\sqrt{1+g^{\prime 2}(x)}}\right|_{x=0} ^{x=x_{0}}+\left.\lambda \eta(x) \frac{f^{\prime}(x)}{\sqrt{1+f^{\prime 2}(x)}}\right|_{x=x_{0}} ^{x=b}
\end{aligned}
$$

the extreme curve has the property that $\left.(d I / d \varepsilon)\right|_{\varepsilon=0}=0$ and since $\eta\left(x_{0}\right)$ is arbitrary. (2.3) implies

$$
\frac{g^{\prime}\left(x_{0}\right)}{\sqrt{1+g^{\prime}\left(x_{0}\right)^{2}}}-\frac{f^{\prime}\left(x_{0}\right)}{\sqrt{1+f^{\prime}\left(x_{0}\right)^{2}}}=0
$$

which is equivalent to

$$
f^{\prime}\left(x_{0}\right)=g^{\prime}\left(x_{0}\right) \text {. }
$$

This proves that $\Gamma$ is tangent to $\Sigma$ at $E$.

The above arguments show that if the extreme curve does exist then it is a curve of (i) or (ii) as described in the lemma. (We do not prove there is a unique extreme curve, in general, this may not be true.) We need to prove existence also.

[Existence]: Let $a$ be the prescribed perimeter and let

$$
\begin{aligned}
& S=\{\Delta(\Gamma) \mid \Gamma \text { joint } P Q \text { in } \Omega, \Gamma=a\} \\
& T=\left\{\Delta(\Gamma) \mid \Gamma \text { joint } P Q \text { in } \Omega, \Gamma=a \text { and } \Gamma \in C^{\infty}\right\}
\end{aligned}
$$

then $T$ is dense in $S$, hence

$$
\sup S=\sup T \text {. }
$$

Let $\Delta_{\infty}=\sup T$, since $T$ is bounded above by $\Omega, \Delta_{\infty}$ is finite and there exists a sequence $\left\{\Delta\left(\Gamma_{n}\right)\right\}$ in $T$ such that $\Delta\left(\Gamma_{n}\right) \uparrow \Delta_{\infty}$. If we can show that $\Delta_{\infty} \in S$, i.e., $\Delta_{\infty}=\Delta\left(\Gamma_{\infty}\right)$ for some curve $\Gamma_{\infty}$ join $P, Q$ in $\Omega$ and $\Gamma_{\infty}=a$ then we are done. 
To this purpose, let each $\Gamma_{n}$ be perimetrized by arc length:

$$
\Gamma_{n}=\left\{\vec{X}_{n}(s)=\left(x_{n}(s), y(s)\right) \mid 0 \leqq s \leqq a\right\}
$$

since $x_{n}^{\prime 2}(s)+y_{n}^{\prime 2}(s)=1 . \quad\left\{x_{n}\right\}$ and $\left\{y_{n}\right\}$ are bounded families of equicontinuous functions, hence by Ascoli's theorem, there exist subsequences $\left\{x_{n_{k}}\right\}$ and $\left\{y_{n_{k}}\right\}$ such that

$$
\begin{gathered}
x_{n_{k}} \longrightarrow x_{\infty} \\
y_{n_{k}}=y_{\infty}
\end{gathered}
$$

uniformly on $[0, a]$ for some $x_{\infty}$ and $y_{\infty} \in C[0, a]$. Let $\vec{X}_{\infty}(s)=$ $\left(x_{\infty}(s), y_{\infty}(s)\right)$ and let $\Gamma_{\infty}=\vec{X}_{\infty}(s)$. Then for any regular partition $0=s_{0}<s_{1}<\cdots<s_{N}=a$ of $[0, a]$, we have

$$
\begin{aligned}
& \sum_{i=1}^{N}\left|\vec{X}_{\infty}\left(s_{i}\right)-\vec{X}_{\infty}\left(s_{i-1}\right)\right| \\
& \leqq \sum_{i=1}^{N}\left|\vec{X}_{\infty}\left(s_{i}\right)-\vec{X}_{n_{k}}\left(s_{i}\right)\right|+\sum_{i=1}^{N}\left|\vec{X}_{n_{k}}\left(s_{i-1}\right)-\vec{X}_{\infty}\left(s_{i-1}\right)\right| \\
& \quad+\sum_{i=1}^{N}\left|\vec{X}_{n_{k}}\left(s_{i}\right)-\vec{X}_{n_{k}}\left(s_{i-1}\right)\right| \\
& \leqq \sum_{i=1}^{N}\left|\vec{X}_{\infty}\left(s_{i}\right)-\vec{X}_{n_{k}}\left(s_{i}\right)\right|+\sum_{i=1}^{N}\left|\vec{X}_{n_{k}}\left(s_{i-1}\right)-\vec{X}_{\infty}\left(s_{i-1}\right)\right|+a
\end{aligned}
$$

Given $\varepsilon>0$, we may choose $n_{k}$ large enough such that

$$
\left|\vec{X}_{\infty}(s)-\vec{X}_{n_{k}}(s)\right|<\frac{\varepsilon}{2 N}
$$

then (2.4) implies

$$
\sum_{i=1}^{N}\left|\vec{X}_{\infty}(s)-\vec{X}_{\infty}\left(s_{i-1}\right)\right|<\varepsilon+a .
$$

Hence $\Gamma_{\infty}$ is rectifiable and $\Gamma_{\infty} \leqq a$. On the other hand, $\Delta\left(\Gamma_{\infty}\right) \geqq \Delta(\Gamma)$ for all $\Delta(\Gamma) \in S$. This implies $\Gamma_{\infty} \geqq a$ and hence $\Gamma_{\infty}=a$.

The following lemma is the main lemma which will be used to prove theorems in the following sections.

Lemma 2.2. Let $P$ and $Q$ be two points in the plane, $\Gamma$ a simple plane curve joining $P$ and $Q$ which encloses with the line segment $\overline{P Q}$ an area $\Delta(\Gamma)$, and $\lambda$ be a constant such that $1 / \lambda \geqq \overline{P Q} / 2$. Then the functional

$$
\varphi(\Gamma)=\lambda \Delta(\Gamma)-\Gamma
$$

achieves its maximum (local) at the smaller circular arc radius $1 / \lambda$. 
Note. There are two circular ares of radius $1 / \lambda$ passing through $P, Q$ (Figure 6). Call the smaller one $\Gamma_{1}$ and the bigger one $\Gamma_{2}$. As we shall see from the following remarks, $\Gamma_{2}$ is a stationary point, not an extreme point of $\varphi$.

Proof. Let $\Gamma(t)=(x(t), y(t)), 0 \leqq t \leqq 1$ be a parametrization of $\Gamma$ with $\Gamma(0)=P, \Gamma(1)=Q$ and $x^{\prime 2}(t)+y^{\prime 2}(t) \neq 0$. Then

$$
\begin{aligned}
\varphi(\Gamma) & =\frac{\lambda}{2} \int_{\partial \Delta} x d y-y d x-\int_{0}^{1} \sqrt{x^{\prime 2}(t)+y^{\prime 2}(t)} d t \\
& =\frac{\lambda}{2} \int_{0}^{1}\left(x y^{\prime}-y x^{\prime}\right) d t-\int_{0}^{1} \sqrt{x^{\prime 2}+y^{\prime 2}} d t \\
& =\int_{0}^{1} F\left(t, x, y, x^{\prime}, y^{\prime}\right) d t
\end{aligned}
$$

where

$$
F\left(t, x, y, x^{\prime}, y^{\prime}\right)=\frac{\lambda}{2}\left(x y^{\prime}-y x^{\prime}\right)-\sqrt{x^{\prime 2}+y^{\prime 2}} .
$$

By the calculus of variations, the extreme curve must satisfy the Euler equations:

$$
F_{x}-\frac{d}{d t} F_{x^{\prime}}=0
$$

and

$$
F_{y}-\frac{d}{d t} F_{y^{\prime}}=0
$$

Computing directly from (2.5) we get

$$
F_{x}=\frac{\lambda}{2} y^{\prime}, \quad F_{x^{\prime}}=-\frac{\lambda}{2} y-\frac{x^{\prime}}{\sqrt{x^{\prime 2}+y^{\prime 2}}}
$$

and

$$
F_{y}=\frac{\lambda}{2} x^{\prime}, \quad F_{y^{\prime}}=\frac{\lambda}{2} y-\frac{y^{\prime}}{\sqrt{x^{\prime 2}+y^{\prime 2}}} .
$$

Substituing (2.8) into (2.6) we have

$$
\lambda y^{\prime}+\frac{d}{d t} \frac{x^{\prime}}{\sqrt{x^{\prime 2}+y^{\prime 2}}}=0
$$

which is equivalent to

$$
\left[\frac{x^{\prime \prime} y^{\prime}-x^{\prime} y^{\prime \prime}}{\left(x^{\prime 2}+y^{\prime 2}\right)^{3 / 2}}-\lambda\right] y^{\prime}=0
$$


Similarly, by substituting (2.9) into (2.7) we have

$$
\left[\frac{x^{\prime \prime} y^{\prime}-x^{\prime} y^{\prime \prime}}{\left(x^{\prime 2}+y^{\prime 2}\right)^{3 / 2}}-\lambda\right] x^{\prime}=0 \text {. }
$$

Since $x^{\prime 2}+y^{\prime 2} \neq 0,(2.10)$ and (2.11) together imply

$$
\frac{x^{\prime \prime} y^{\prime}-x^{\prime} y^{\prime \prime}}{\left(x^{\prime 2}+y^{\prime 2}\right)^{3 / 2}}=\lambda \text {. }
$$

The left hand side of (2.12) represents the curvature of $\Gamma$. Thus $\Gamma$ is a curve of constant curvature $\lambda$ and therefore a circular arc of radius $1 / \lambda$. There are two such circular arcs (Figure 7 ), say $\Gamma_{1}$ (the smaller one) and $\Gamma_{2}$ (the bigger one). To show that $\phi$ achieves its maximum on $\Gamma_{1}$, one can write down the second variation and show it is negative at $\Gamma_{1}$, however it is a tedious task. The following method seems much easier.

Fixing the arc length $\Gamma$, the classical isoperimetric inequality implies that $\Delta(\Gamma)$, and hence $\varphi(\Gamma)=\lambda \Delta(\Gamma)-\Gamma$, will achieve its maximum when $\Gamma$ is a circular arc. Thus to maximize $\varphi(\Gamma)$, it suffices to consider the value of $\varphi$ on the family of circular arcs passing through $P$ and $Q$. Now let $\Gamma$ be a circular arc in a neighborhood of $\Gamma_{1}$ with radius $r$ and center 0 . Let $\theta=(1 / 2) \angle P O Q$ and let $a=$ $(1 / 2) \overline{P Q}$ (Figure 7). Then we have

$$
\begin{aligned}
\Gamma & =2 r \theta \\
\Delta & =r^{2} \theta-a r \cos \theta \\
\theta & =\sin ^{-1} \frac{a}{r}
\end{aligned}
$$

and

$$
\begin{gathered}
\varphi=\varphi(r)=\lambda \Delta(r)-\Gamma(r) \\
=\left(\lambda r^{2}-2 r\right) \sin \frac{a}{r}-\lambda a \sqrt{r^{2}-a^{2}} \\
\varphi^{\prime}(r)=2(\lambda r-1)\left(\sin ^{-1} \frac{a}{r}-\frac{a}{\sqrt{r^{2}-a^{2}}}\right) \\
\varphi^{\prime \prime}(r)=2 \lambda\left(\sin ^{-1} \frac{a}{r}-\frac{a}{\sqrt{r^{2}-a^{2}}}\right) \\
+2(\lambda r-1)\left[\frac{-1}{\sqrt{r^{2}-a^{2}}} \frac{a}{r}+\frac{a r}{\left(r^{2}-a^{2}\right)^{3 / 2}}\right] \\
\varphi^{\prime \prime}\left(\Gamma_{1}\right)=\varphi^{\prime \prime}\left(\frac{1}{\lambda}\right) \\
=2 \lambda\left(\sin ^{-1} a \lambda-\frac{a \lambda}{\sqrt{1-(a \lambda)^{2}}}\right) .
\end{gathered}
$$


By assumption $1 / \lambda>(1 / 2) \overline{P Q}=a$, so $0<a \lambda<1$. If we introduce the angle $\alpha=\sin ^{-1} a \lambda$, then $0<\alpha<\pi / 2$ and $a \lambda / \sqrt{1-(a \lambda)^{2}}=\tan \alpha$. (2.16) becomes

$$
\phi^{\prime \prime}\left(\Gamma_{1}\right)=2 \lambda(\alpha-\tan \alpha)<0 .
$$

This proves that $\varphi\left(\Gamma_{1}\right)$ is a local maximum.

\section{REMARK.}

(i) Equation (2.14) can be written as

$$
\phi^{\prime}(r)=2(\lambda r-1)(\theta-\tan \theta), \quad 0 \leqq \theta \leqq \frac{\pi}{2} .
$$

Thus $\phi^{\prime}(r)=0$ if and only if $r=1 / \lambda$ or $\theta=\tan \theta$; the second case can happen only if $\theta=0$ and hence $\Gamma$ would be the line segment $\overline{P Q}$, which is not a candidate for an extremal, as we have seen from the first part of the proof. Hence we obtain again that the circular arcs of radius $1 / \lambda$ are extremal curves of $\varphi$.

(ii) $\varphi^{\prime}(r)>0$ if $a<r<1 / \lambda$. This means that $\varphi$ is strictly increasing as $r$ varies from $a$ to $1 / \lambda$ or geometrically, $\varphi\left(\Gamma_{1}\right)>\varphi(\Gamma)$ for all smaller circular arcs $\Gamma$ lying "above" $\Gamma_{1}$ (Figure 8).

(iii) $\varphi^{\prime}(r)<0$ if $r>1 / \lambda$. This means that $\varphi$ is strictly decreasing as $r$ increases from $1 / \lambda$ to $\infty$, or geometrically, $\varphi\left(\Gamma_{1}\right)>\varphi\left(\Gamma^{*}\right)$ for all circular arcs lying between $\Gamma_{1}$ and $\overline{P Q}(r=\infty)$ (Figure 8).

(iv) $\Gamma_{2}$ is a stationary curve but not an extreme curve.

For let $C$ be any bigger circular arc which lies "inside of" $\Gamma_{2}$, that is $r$ (radius of $C$ ) $<1 / \lambda$; and let $P^{\prime}$ and $Q^{\prime}$ be any two points on $C$ such that the subarc $C_{P^{\prime} Q^{\prime}}$ of $C$ between $P^{\prime}$ and $Q^{\prime}$ is a smaller circular arc (Figure 9). Now let $C_{P^{\prime} Q^{\prime}}^{*}$ be the small circular arc of radius $1 / \lambda$ passing through $P^{\prime}$ and $Q^{\prime}$. Applying our lemma to $C_{P^{\prime} Q^{\prime}}$ and $C_{P^{\prime} \ell^{\prime}}^{*}$ we have:

$$
\lambda \Delta\left(C_{P^{\prime} Q^{\prime}}\right)-C_{P^{\prime} Q^{\prime}}<\lambda \Delta\left(C_{P^{\prime} Q^{\prime}}^{*}\right)-C_{P^{\prime} Q^{\prime}}^{*} .
$$

Let $S$ be the shaded area as shown in Figure 9. Adding the quantiy $\lambda S-C_{P P^{\prime}}-C_{Q^{\prime} Q}$ to both sides of (2.18) we have

$$
\mathscr{P}(C)=\lambda \Delta(C)-C \leqq \lambda \Delta\left(C^{*}\right)-C^{*}=\varphi\left(C^{*}\right)
$$

where $C^{*}=C_{P P^{\prime}}+C_{P^{\prime} Q^{\prime}}^{*}+C_{Q^{\prime} Q}$.

Now if we let $C_{1}$ be the circular arc through $P$ and $Q$ which has the same length as $C^{*}$, then by the classical isoperimetric inequality we have

$$
\varphi(C)<\varphi\left(C^{*}\right)<\varphi\left(C_{1}\right)
$$


By continuing this process, one will increase $\varphi$ until $C_{1}$ shrink to $\Gamma_{1}$.

On the other hand, since $C$ can be chosen to be very close to $\Gamma_{2}$ and $C_{1}$ can be made very close to $C$ (by taking $P^{\prime}$ close enough to $Q^{\prime}$ ), we have

$$
\varphi\left(\Gamma_{2}\right)<\varphi\left(C_{1}\right)
$$

by the continuity of $\varphi$.

If $C$ is a bigger circular arc lying "outside of" $\Gamma_{2}$, by using the same argument as above, we will obtain the same result as (2.19). However this time $C_{1}$ is not a shrunken circular arc of $C$, but an enlarged circular arc of $C$. As this process continues, $C_{1}$ will grow bigger and bigger and $\varphi$ will go to $\infty$, since $\lambda \Delta-\Gamma \sim \lambda 0\left(r^{2}\right)-$ $0(r) \rightarrow \infty$, as $r \rightarrow \infty$.

The above arguments show that $\varphi\left(\Gamma_{2}\right)$ is a minimum among the family of circular arcs. Obviously, it is not a minimum among all curves, since $\varphi(\Gamma)<\varphi\left(\Gamma_{2}\right)$ for noncircular curves $\Gamma$ which have the same arc length as $\Gamma_{2}$.

The following lemma is an immediate result of Lemma 2.2.

LEMMA 2.3. Let $\Lambda$ be a plane curve joining $P$ and $Q$, which does not meet the smaller circular arc $C$ of radius $1 / \lambda . \quad \lambda>(1 / 2) \overline{P Q}$, and let $\Gamma$ be any simple plane curve joining $P$ and $Q$ which encloses an area $\Delta(\Gamma)$ with $\Lambda$. Then the functional

$$
\varphi(\Gamma)=\lambda \Delta(\Gamma)-\Gamma
$$

achieves its maximum on $C$.

3. Some results of Concus, Finn and Giusti. The following theorem of maximal principle is proved by Concus and Finn [3].

Theorem 3.1. (Maximal principle). Let $\Omega$ be a plane domain with piecewise smooth boundary $\Sigma$, and let $u, v$ be two functions in $C^{2}(\Omega)$. Suppose that

$$
\begin{gathered}
\operatorname{div} T u \leqq \operatorname{div} T v \quad \text { in } \quad \Omega \\
T u \cdot \nu \geqq T v \cdot \nu \text { on } \quad \Sigma-\Sigma_{0}
\end{gathered}
$$

where $\Sigma_{0}$ denotes the set of points in $\Sigma$ at which the normal is not defined. Then $u \equiv v+$ constant in $\Omega$.

Let $\Omega$ be as above and consider the following capillary free surface equations

$$
\operatorname{div} T u=H \quad \text { in } \Omega, \quad T u \cdot \nu=\cos \gamma \quad \text { on } \quad \Sigma-\Sigma_{0},
$$


where $H$ and $\gamma$ are constants, and $\nu$ denotes the outer normal of $\Sigma$. Theorem 3.1 implies that the solution of (3.1) is unique up to an additive of constant if it exists.

The following theorems for existence is proved by Giusti [13] and [15].

THEOREM 3.2. Suppose that there exists an $\varepsilon>0$ such that

$$
\left|\frac{\Sigma^{*}}{\Sigma}-\frac{\Omega^{*}}{\Omega}\right| \cos \gamma<(1-\varepsilon) \frac{\Gamma}{\Sigma}
$$

for all curves $\Gamma$ in $\Omega$ (Figure 2). Then (3.1) has a solution.

THEOREM 3.3. Let $\Omega$ be a convex domain in the plane and let $k(p)$ be the curvature of $\Sigma$ at $p$. Suppose that

$$
k(p) \leqq \frac{\Omega}{\Sigma}
$$

for all $p \in \Sigma$, then (3.1) has a solution for all $\gamma$.

THEOREM 3.4. Suppose that there holds

$$
\left|\frac{\Sigma^{*}}{\Sigma}-\frac{\Omega^{*}}{\Omega}\right|<\frac{\Gamma}{\Sigma}
$$

for all $\Omega^{*} \neq \varnothing$ and $\Omega$, then (3.1) has a solution for $\gamma=0$.

4. Geometric condition for the existence of solutions. In this section we give a geometric condition on $\Omega$, which guarantees the existence of solutions of (3.1) for all contact angles $\gamma$.

DEFINITION 4.1. A plane domain $\Omega$ with piecewise smooth boundary has the interior rolling disk property of radius $R$ if:

(i) for any regular point $P$ on $\Sigma$, one can contact a circle of radius $R$ inside $\Omega$ so that no antipodes of the circle lie on $\Sigma$.

(ii) for irregular points $P$ on $\Sigma$, one can contact a circle of radius $R$ inside $\Omega$ in any direction between $\nu(P+)$ and $\nu(P-)$ so that no antipodes of the circle lie on $\Sigma$, where $\nu(P+)$ and $\nu(P-)$ denotes the right hand and left hand normals of $\Sigma$ at $P$.

Roughly speaking, a plane domain $\Omega$ has the interior rolling disk property of radius $R$ if one can rotate a disk of radius $R$ along every point of $\Sigma$ so that no antipodes of the disk lie on $\Sigma$. Thus a triangle does not have the interior rolling disk property for any $R$. A domain with a narrow aperture fails to have the property if $R$ is bigger 
than half of the aperture.

We now state our main results.

THEOREM 4.1. If $\Omega$ has the interior rolling disk property of radius $R=\Omega / \Sigma$, then (3.1) has a solution for any contact angle $\gamma$.

THEOREM 4.2. Let $\Gamma_{1}, \Gamma_{2}, \cdots$, be all of the circular arcs of radius $R=\Omega / \Sigma$ in $\Omega$ with end points on $\Sigma$ which are tangent to $\Sigma$ at regular points of $\Sigma$ and contact to $\Sigma$ at singular points of $\Sigma$. If there holds that

$$
\left|\frac{\Sigma_{i}^{*}}{\Sigma}-\frac{\Omega_{i}^{*}}{\Omega}\right| \leqq \frac{\Gamma_{i}}{\Sigma}
$$

for all $i=1,2, \cdots$, then (3.1) has a solution for all contact angle $\gamma$.

The proof of the above theorems is based on the following idea. Suppose we can show that

$$
\left|\frac{\Sigma^{*}}{\Sigma}-\frac{\Omega^{*}}{\Omega}\right| \leqq \frac{\Gamma}{\Sigma}
$$

for all possible curve $\Gamma$ in $\Omega$, then for any $\gamma$ with $0<\gamma \leqq \pi / 2$, Concus-Finn-Giusti's condition (3.2) holds by taking $\varepsilon=1-\cos (\gamma / 2)$ and hence a solution exists.

However (4.3) is equivalent to

$$
-\Gamma+\frac{1}{R} \Omega^{*} \leqq \Sigma^{*} \leqq \Gamma+\frac{1}{R} \Omega^{*}
$$

where $R=\Omega / \Sigma$.

Let $\Sigma^{*}$ be fixed and let $P, Q$ be its end points on $\Sigma$, consider the functionals

$$
f(\Gamma)=\Gamma+\frac{1}{R} \Omega^{*}(\Gamma)
$$

and

$$
g(\Gamma)=-\Gamma+\frac{1}{R} \Omega^{*}(\Gamma)
$$

defined on all curves $\Gamma$ in $\Omega$ which join $P$ and $Q$. If we can show that

$$
\operatorname{Max}_{\Gamma} g(\Gamma) \leqq \Sigma^{*} \leqq \operatorname{Min}_{\Gamma} f(\Gamma)
$$

for all $P, Q \in \Sigma$, then we are done. 
On the other hand, let $\Omega^{\prime}=\Omega-\Omega^{*}, \Sigma^{\prime}=\Sigma-\Sigma^{*}$ then

$$
\begin{aligned}
f(\Gamma) & =\Gamma+\frac{1}{R}\left(\Omega-\Omega^{\prime}\right) \\
& =\frac{1}{R} \Omega-\left(-\Gamma+\frac{1}{R} \Omega^{\prime}\right) \\
& =\Sigma-\left(-\Gamma+\frac{1}{R} \Omega^{\prime}\right)
\end{aligned}
$$

thus minimizing $f(\Gamma)$ is equivalent to maximizing $-\Gamma+(1 / R) \Omega^{\prime}$, which is just maximizing $g(T)$ with $\Sigma^{*}$ replaced by $\Sigma^{\prime}$ and $\Omega^{*}$ replaced by $\Omega^{\prime}$. Therefore the only thing we need to do is to maximize $g$.

We need only consider the case $\Gamma \leqq \Sigma^{\prime}$, for if $\Gamma>\Sigma^{\prime}$

$$
g(\Gamma)=-\Gamma+\frac{1}{R} \Omega^{*}<-\Sigma^{\prime}+\frac{1}{R} \Omega=g\left(\Sigma^{\prime}\right) .
$$

Now let $\Gamma$ be any curve with $d(P, Q) \leqq I^{\prime} \leqq \Sigma^{\prime}$ where $d(P, Q)$ is the distance between $P$ and $Q$. By Lemma 2.1, among all curves of arc length $\Gamma, \Omega^{*}$ and thus $g(\Gamma)$ would achieve its maximum in one of the following cases:

(i) a circular are if one can draw a circular arc in $\Omega$ with arc length $\Gamma$ (3.8).

(ii) a curve which coincides with some subarcs of $\Sigma$ and circular arcs of equal radii with the property that if the end points of the circular arcs are smooth points of $\Sigma$ then the circular arcs are tangent to $\Sigma(3.9)$.

Let

$$
\mathscr{F}=\left\{\Gamma \mid d(P, Q) \leqq \Gamma \leqq \Sigma^{\prime}, \Gamma \text { is a curve satisfying (3.8) or (3.9) }\right\} \text {. }
$$

Then we have

$$
\underset{\text { any } \Gamma}{\operatorname{Max}} g(\Gamma)=\operatorname{Max}_{\Gamma^{\prime}} g(\Gamma) .
$$

This reduces the maximization over a very complicated class of curves to one over a much simpler class.

To prove Theorem 4.1, we shall show that $g$ achieves its maximum on the boundary $\Sigma^{\prime}$ and since

$$
g\left(\Sigma^{\prime}\right)=-\Sigma^{\prime}+\frac{1}{R} \Omega=-\Sigma^{\prime}+\Sigma=\Sigma^{*}
$$

(4.4) hold for $\Sigma^{\prime}$ and hence will hold for all possible curve.

Before proving the theorems, let us define some notation: Let $C$ be a simple closed curve, the interior of which is divided by $\Sigma$ 
into subregions $\Delta_{1}, \Delta_{2}, \cdots, \Delta_{k}$, we will call $\Delta_{i}$ positive $(+)$ if $\Delta_{2}$ lies inside $\Omega$, negative (-) if $\Delta_{i}$ lies outside $\Omega$.

We need the following lemmas.

Lemma 4.3. Let $\Omega$ be as in Theorem 4.1; then no circle of radius less than $R$ can have its interior divided by $\Sigma$ into subregions containing more than one negative region.

Proof. Suppose there exists a circle $S_{r}(0)$ of radius $r \leqq R$ centered at 0 , which is divided by $\Sigma$ into more than one negative regions, let $\Delta_{1}, \Delta_{2}, \Delta_{3}$ be three adjacent regions of the type -, +, - and let $\Sigma_{A B}, \Sigma_{C D}$ be two of the subarcs of $\Sigma$ bounding $\Delta_{2}$ (Figure 10). Shrink $S_{r}(0)$ with respect to 0 until the first point of contact (with $\Sigma_{A B} \cup \Sigma_{C D}$ ) happens, say at $P \in \Sigma_{C D}$, i.e., there exists a circle $S_{t}(0), t \leqq r \leqq R$, which contacts $\Sigma_{C D}$ at $P$ and intersects $\Sigma_{A B}$ at some points, say $A^{\prime}, B^{\prime}$. Now take the circle $S_{R}$ of radius $R$ containing $S_{t}(0)$ and tangent to $S_{t}(0)$ at $P . \quad S_{R}$ contacts $\Sigma$ at $P$ but does not lie inside $\Omega$, contradicting the hypothesis that $\Omega$ has the interior rolling disk property of radius $R$.

Definition 4.2. A subregion $\Omega^{\prime} \subset \Omega$ is convex in $\Omega$ if for every two points $P, Q \in \Omega^{\prime}$, the line segment $\overline{P Q} \subset \Omega$ implies $\overline{P Q} \subset \Omega^{\prime}$.

Lemma 4.4. Let $\Omega$ be as in Theorem 4.1 and $\Gamma$ be a circular arc of radius $R$ in $\bar{\Omega}$ which is not contained in $\Sigma$, and let the subregion $\Omega^{*}$ be convex in $\Omega$. Then

$$
g(\Gamma)<g\left(\Gamma^{\prime}\right) \quad(\text { strictly }) .
$$

Proof. Let $\Gamma^{\prime}$ be the completing circular arc of $\Gamma$. Then by Lemma 4.3 , either $\Sigma^{*}$ or $\Sigma^{\prime}$ lies inside the circle $\Gamma \cup \Gamma^{\prime}$.

If $\Sigma^{*}$ lies inside the circle $\Gamma \cup \Gamma^{\prime}$, then $\Omega^{*} \leqq \pi R^{2}$, and there are two possible cases $\Sigma^{*}>\Gamma^{\prime}$ or $\Sigma^{*} \leqq \Gamma^{\prime}$.

(i) If $\Sigma^{*}>\Gamma^{\prime}$ (Figure 11a), then

$$
\frac{1}{R} \Omega^{*} \leqq \frac{1}{R} \pi R^{2}=\pi R<2 \pi R=\Gamma+\Gamma^{\prime}<\Gamma+\Sigma^{*}
$$

hence we have

$$
g(\Gamma)=-\Gamma+\frac{1}{R} \Omega^{*}<\Sigma^{*}=g\left(\Sigma^{\prime}\right) .
$$

(ii) If $\Sigma^{*} \leqq \Gamma^{\prime}$ (Figure 11b), then by the isoperimetric inequality $\frac{1}{R} \Omega^{*} \leqq \frac{1}{R} \frac{\left(\Sigma^{*}+\Gamma\right)^{2}}{4 \pi} \leqq \frac{\Gamma^{\prime}+\Gamma}{4 \pi R} \cdot\left(\Sigma^{*}+\Gamma\right)=\frac{2 \pi R}{4 \pi R}\left(\Sigma^{*}+\Gamma\right)<\Sigma^{*}+\Gamma$. 
We still have

$$
g(\Gamma)=-\Gamma+\frac{1}{R} \Omega^{*}<\Sigma^{*}=g\left(\Sigma^{\prime}\right) .
$$

If $\Sigma^{\prime}$ lies inside the circle $\Gamma \cup \Gamma^{\prime}$ (Figure 11c), then by Lemma $2.3 \Sigma^{\prime} \leqq-\Gamma+(1 / R) \Omega^{\prime}$, hence we have

$$
\begin{aligned}
g(\Gamma) & =-\Gamma+\frac{1}{R} \Omega^{*}=-\Gamma+\frac{1}{R}\left(\Omega-\Omega^{\prime}\right) \\
& =\Sigma-\Gamma-\frac{1}{R} \Omega^{\prime}<\Sigma+\Gamma-\frac{1}{R} \Omega^{\prime} \\
& =\Sigma-\left(-\Gamma+\frac{1}{R} \Omega^{\prime}\right) \leqq \Sigma-\Sigma^{\prime} \\
& =\Sigma^{*}=g\left(\Sigma^{\prime}\right) .
\end{aligned}
$$

This proves the lemma.

Lemma 4.5. Let $\Gamma$ be a curve in $\bar{\Omega}$ whose end points $P$ and $Q$ lie on $\Sigma$, let $\Omega^{\prime}$ and $\Omega^{*}$ be the two disjoint regions cut by $\Gamma$. Suppose that one can draw a piecewise smooth curve $\Gamma_{1}$ in $\bar{\Omega}^{\prime}$ which join $P$ and $Q$ and with the property that a circle of radius $R$ can be rotated on it in such a way that the circular arc between $\Gamma$ and $\Gamma_{1}$ is always a smaller circular arc of the circle (Figure 12). Then

$$
g(\Gamma)<g\left(\Gamma_{1}\right) .
$$

Proof. Let $\Omega_{1}^{\prime}$ and $\Omega_{1}^{*}$ be the corresponding subregions cut by $\Gamma_{1}$ such that $\Omega_{1}^{\prime} \subset \Omega^{\prime}$ and $\Omega_{1}^{*} \supset \Omega^{*}$. Let $A$ and $B$ be two points on $\Gamma_{1}$ which are close enough such that the two circles of radius $R$ contact to $\Gamma_{1}$ at $A$ and $B$ from the side of $\Omega_{1}^{*}$ have nonempty intersection in $\Omega_{1}^{*}-\Omega^{*}$. Let $C$ be the intersection of these two circles and let $D, E, F, G$ be the intersections of them with $\Gamma$ as shown in Figure 12. Let $S, \Delta_{A}, \triangle$ be the regions enclosed by $A B, B C, C A$; $D A F, F D ; F G, G B C, C F$ respectively, and consider the new curve $\Gamma(A), \Gamma(A B)$ defined as follows

$$
\begin{aligned}
\Gamma(A) & =P D+D A+A F+F Q \\
\Gamma(A B) & =P D+D A+A B+B G+G Q .
\end{aligned}
$$

Here we simplify the notation by writing $P D=\Gamma_{P D}$ (the restriction of $\Gamma$ between $P$ and $D$ ), $D A=$ circular arc between $D$ and $A, A B=\Gamma_{1 A B}$ (the restriction of $\Gamma_{1}$ between $A$ and $B$ ) $\cdots$ etc. Then

$$
g(\Gamma(A))-g(\Gamma)=-D A-A F+D F+\frac{1}{R} \Delta_{A}
$$




$$
\begin{aligned}
& =\left(\frac{1}{R} \Delta_{A}-D A F\right)-\left(\frac{1}{R} \cdot 0-D F\right) \\
& =\varphi(D A F)-\varphi(D F) \geqq 0
\end{aligned}
$$

(Lemma 2.3 with $\Lambda=D F$ )

$$
\begin{aligned}
g(\Gamma(A B)) & -g(\Gamma(A)) \\
= & -A B-B G+A F+F G+\frac{1}{R}(\Delta+S) \\
= & -A B-B G+(A C+C F)+F G+\frac{1}{R}(\Delta+S) \\
= & (A C+B C-A B)+\frac{1}{R} S+\left(\frac{1}{R} \Delta-B C-B G+C F+F G\right) \\
= & (A C+B C-A B)+\frac{1}{R} S+\left[\frac{1}{R} \Delta-C B G-\left(\frac{1}{R} \cdot 0-C F G\right)\right] \\
= & A C+B C-A B+\frac{1}{R} S+[\varnothing(C B G)-\varphi(C F G)] \\
\geqq & A C+B C-A B,
\end{aligned}
$$

since $(1 / R) S \geqq 0$ and $\varphi(C B G) \geqq \varphi(C F G)($ Lemma 2.3 with $\Lambda=C F G)$.

Suppose that we can show that

$$
A C+B C>A B
$$

provided $A$ and $B$ are sufficiently close, then

$$
g(\Gamma(A B)) \geqq g(\Gamma(A))
$$

and using this together with (4.8) and the standard compactness argument we obtain $g\left(\Gamma_{1}\right)>g(\Gamma)$.

Let $k(A)$ be the curvature of $\Gamma_{1}$ at $A$, since $\Gamma_{1}$ can be rotated on it form the side of $\Omega_{1}^{*}$ a circle of radius $R$, we have $k(A) \leqq 1 / R$ provided that $A$ is a regular point of $\Gamma_{1}$. If $A$ is not a regular point of $\Gamma_{1}$, then by hypothesis, we may consider $k\left(A^{+}\right)$and $k\left(A^{-}\right)$, the curvatures of the two smooth curves meeting at $A$, we still have $k\left(A^{+}\right) \leqq 1 / R$ and $k\left(A^{-}\right) \leqq 1 / R$. We shall prove (4.9) under these curvature conditions. We consider here only the case where $A$ is a regular point of $\Gamma_{1}$; for nonregular points, essentially the same arguments work.

Case (i ). $|k(A)|<1 / R$.

There exists a neighborhood $N(A)$ of $A$ on $\Gamma_{1}$ on which $|k(B)|<$ $1 / R$ for all $B \in N(A)$. Pick a point $B \in N(A)$ such that the two circles 
of radius $R$ contact to $\Gamma_{1}$ at $A$ and $B$ from the side of $\Omega_{1}^{*}$ have nonempty intersection, and let $C$ be as above. Let $(O, X, Y)$ be a local coordinate system centered at $O \in \Gamma_{1}$ with $O$ between $A$ and $B$ in which $O X$ and $O Y$ coincide with the tangent and normal directions of $\Gamma_{1}$ at $O$ respectively. As $O$ moves from $A$ to $B, C$ will first lie on one side of $O Y$ and then on the other side. Since $\Gamma_{1}$ is smooth between $A$ and $B$ there exists a point $O$ between $A$ and $B$ at which $O Y$ passes through $C$ (Figure 13). Let $A O$ and $A C$ be expressed in this coordinate system as $y(x)$ and $z(x)$ respectively, and let $a, b$ be the $x$-axis components of $A, B$ respectively. Then we have

$$
y(a)=z(a), \quad y^{\prime}(a)=z^{\prime}(a), \quad y^{\prime}(0)=0
$$

the curvature condition gives

$$
-\frac{d}{d x} \frac{z^{\prime}}{\sqrt{1+z^{\prime 2}}} \leqq \frac{d}{d x} \frac{y^{\prime}}{\sqrt{1+y^{\prime 2}}} \leqq \frac{d}{d x} \frac{z^{\prime}}{\sqrt{1+z^{\prime 2}}} .
$$

Integrate (4.11) from $a$ to $x$ and use the initial condition $y^{\prime}(a)=z^{\prime}(a)$ we get

$$
-\frac{z^{\prime}(x)}{\sqrt{1+z^{\prime 2}(x)}} \leqq \frac{y^{\prime}(x)}{\sqrt{1+y^{\prime 2}(x)}} \leqq \frac{z^{\prime}(x)}{\sqrt{1+z^{\prime 2}(x)}}
$$

for all $a \leqq x \leqq 0$; this is equivalent to

$$
y^{\prime 2}(x) \leqq z^{\prime 2}(x)
$$

and hence

$$
A C=\int_{a}^{0} \sqrt{1+z^{\prime 2}(x)} d x \geqq \int_{a}^{0} \sqrt{1+y^{\prime 2}(x)} d x=A 0 .
$$

Similarly, $B C \geqq B O$ and we get

$$
A C+B C \geqq A B \text {. }
$$

Case (ii). $k(A) \leqq-1 / k$ (this case may happen on $\Sigma$ ).

There exists a neighborhood $N(A)$ of $A$ on $\Gamma_{1}$ on which $k(B)<0$ for all $B \in N(A)$. Pick a point $B \in N(A)$ and set up the coordinate system $(O, X, Y)$ as in Case (i) (Figure 14), and let $y(x), z(x), a, b$ be defined in this coordinate system as in Case (i). Then we have

$$
y^{\prime}(a)=z^{\prime}(a), \quad y^{\prime}(0)=0 .
$$

Since $y$ has negative curvature for all $a \leqq x \leqq 0, y^{\prime \prime}(x)<0$ for all $a \leqq x \leqq 0$. That is, $y^{\prime}$ is strictly decreasing on $[a, 0]$. Similarly, $z$ has positive curvature for all $a \leqq x \leqq 0, z^{\prime}$ is strictly increasing on $[a, 0]$. 
Thus for $x \in[a, 0]$ we have

$$
z^{\prime}(x)>z^{\prime}(a)=y^{\prime}(a)>y^{\prime}(x)>y^{\prime}(0)=0
$$

which implies that $z^{\prime 2}(x)>y^{\prime 2}(x)$ and we have

$$
A C=\int_{a}^{0} \sqrt{1+z^{\prime 2}(x)} d x>\int_{a}^{0} \sqrt{1+y^{\prime 2}(x)} d x=A O .
$$

Similarly $B C>B O$ and we obtain

$$
A C+B C>A B \text {. }
$$

This completes the proof.

REMARK. The lemma still is true if we replace "always a smaller circular arc" by "not a bigger circular arc" i.e., we allow semi-circles to happen between $\Gamma$ and $\Gamma_{1}$. As we can take a sequence of curves $\left\{\Gamma_{1, n}\right\}$ satisfies the hypothesis of the lemma, such that

$$
\begin{aligned}
& \Gamma_{1, n} \longrightarrow \Gamma_{1} \quad \text { (in arc length) } \\
& \Omega_{1, n}^{*} \longrightarrow \Omega_{1}^{*}
\end{aligned}
$$

then

$$
g(\Gamma)<\lim _{n \rightarrow \infty} g\left(\Gamma_{1, n}\right)=g\left(\Gamma_{1}\right)
$$

Lemma 4.6. Let $\Omega$ be as in Theorem 4.1 and $\Gamma$ be a circular arc of radius $>R$. Then

$$
g(\Gamma)<g\left(\Sigma^{\prime}\right)
$$

still holds.

Proof. Let $P, Q \in \Sigma$ be the end points of $\Gamma$. There are two cases to consider:

(i) $d(P, Q) \leqq 2 R$ and $\Gamma$ is not a bigger circular arc. In this case, one can draw a nonbigger circular arc $\Gamma_{1}$ of radius $R$ passing through $P, Q$ and lying "outside" $\Gamma$ (i.e., $\Gamma_{1} \subset \Omega^{\prime}=\Omega-\Omega^{*}$ ). By the remark of Lemma 4.5 and Lemma 4.4 we have

$$
g(\Gamma)<g\left(\Gamma_{1}\right)<g\left(\Sigma^{\prime}\right) .
$$

(ii) $d(P, Q)>2 R$ or $\Gamma$ is a bigger circular arc. Let $\Omega^{\prime}=\Omega-\Omega^{*}$, let $\Gamma_{t}$ be any curve joining $P, Q$ in $\bar{\Omega}^{\prime}$ with the property that a circle of radius $R$ can be rotated on it such that the circular arc of the circle which lies between $\Gamma_{t}$ and $\Gamma$ is not a bigger circular arc. Let $\Omega_{t}^{*}$. be the region bounded by $\Gamma_{t}$ and $\Sigma^{*}$, and let $\Omega_{t}^{\prime}=\Omega-\Omega_{t}^{*}$. Consider the set $S_{1}=\left\{\Omega_{t}^{*}\right\}$, partially order $S_{1}$ by set inclusion. It is 
easy to see that $S_{1}$ is closed in every linear ordered subset, i.e., every linear ordered subset is bounded above by an element in $S_{1}$. Hence, by Zorn's lemma, there exists a maximal element $\Omega_{1}^{*}$ in $S_{1}$. Since $\Gamma$ is a curve of curvature $<R, S_{1}$ is nonempty, and we have $\Omega_{1}^{*} \supset \Omega^{*}$ strictly. (Actually, $\Omega_{1}^{*}=\Omega^{*}$ if and only if $\Gamma$ is a circular arc of radius $R$ or $\Gamma=\Sigma^{\prime}$.) Let $\Gamma_{1}=\partial \Omega_{1}^{*} \cap \Omega$, by the remark of Lemma 4.5 , we have

$$
g(\Gamma)<g\left(\Gamma_{1}\right) .
$$

In other words, $g$ can be increased by "moving $\Gamma$ towerd $\Sigma$ " to a new curve $\Gamma_{1}$ in a certain distance which depends on the situation of $\Gamma$ and the geometry of $\Sigma$.

Now repeat the argument on $\Gamma_{1}$. (The argument can be repeated if a circle of radius $R$ can be rotated on it from the side of $\Omega_{1}^{*}$. It is clear that $\Gamma_{1}$ has this property from the construction of $\Gamma_{1}$.) We obtain a curve $\Gamma_{2}$ and a subregion $\Omega_{2}^{*} \supset \Omega_{1}^{*}$ such that

$$
g\left(\Gamma_{1}\right) \leqq g\left(\Gamma_{2}\right) .
$$

Continue this process we get a monotone sequence of curves $\Gamma_{n}$ and an increasing sequence of regions $\Omega_{n}^{*}$ such that

$$
g\left(\Gamma_{n}\right) \leqq g\left(\Gamma_{n+1}\right) .
$$

Since $\Omega_{n}^{*} \subset \Omega_{n+1}^{*} \subset \Omega$ and $\Omega$ is bounded we have $\lim _{n \rightarrow \infty} \Omega_{n}^{*}=$ $\Omega_{\infty}^{*} \cap \Omega$. Let $\Gamma_{\infty}=\partial \Omega_{\infty}^{*} \cap \Omega^{\prime}$; by our construction of $\Gamma_{n}$, we have $\lim _{n \rightarrow \infty} \Gamma_{n}=\Gamma_{\infty}$ uniformly in pointwise convergence and also converges in the corresponding arc length. Hence

$$
g\left(\Gamma_{1}\right)<\lim _{n \rightarrow \infty} g\left(\Gamma_{n}\right)=g\left(\Gamma_{\infty}\right) .
$$

We claim that $\Gamma_{\infty}=\Sigma^{\prime}$.

Let $E=\Gamma_{\infty}-\left(\Gamma_{\infty} \cap \Sigma^{\prime}\right)=\bigcup_{i=1}^{\infty} E_{i}$, where each $E_{i}$ is a connected component of $E$. Since $\Gamma_{\infty}$ is the monotone limit of $\Gamma_{n}$ and each $\Gamma_{n}$ has the property that a circle of radius $R$ can be contacted at each point of it from the side of $\Omega_{n}^{*}, \Gamma_{n}$ inherits the property too, and so does $E_{i}$ for each $i$.

If $E_{i} \neq \varnothing$ and is not a circular arc of radius $R$, then there exists a point $x \in E_{i}$ with the property that a circle $B(R)$ of radius $R$ can be contacted on it from the side of $\Omega_{\infty}^{*}$ such that $E_{i} \cap B(R)=\{x\}$. Thus, it is possible to move $B(R)$ across $E_{i}$ slightly so that $B(R) \cap \Omega_{\infty}^{\prime}$ is a smaller circular arc of $B(R)$, (Figure 15). Since $\Gamma_{n} \rightarrow \Gamma_{\infty}$ uniformly, there exists $\Gamma_{n-1}$ such that $B(R) \cap \Omega_{n-1}^{\prime}$ is also a smaller circular arc of $B(R)$. Let $\{A, B\}=B(R) \cap \Gamma_{n}, \overparen{A B}=B(R) \cap \Omega_{n}^{\prime}$, $\Gamma_{n, t}=\Gamma_{n}-\Gamma_{n \mid A B}+\overparen{A B}$, and $\Omega_{n, t}^{*}$ be the region bounded by $\Gamma_{n, t}$ and 
$\Sigma^{*}$. We have $\Omega_{n t}^{*} \supset \Omega_{n}^{*}$ and $\Gamma_{n, t}$ has the property that a circle of radius $R$ can be contacted at each point of it such that the circular are between $\Gamma_{n}$ and $\Gamma_{n-1}$ is always a smaller circular are of the circle. This contradicts the maximality of $\Omega_{n}^{*}$.

Thus, each $E_{i}$ should be a circular arc of radius $R$ or an empty set. The first case is impossible since $\Omega$ has the interior rolling disk property of radius $R$. We conclude that $E_{i}=\varnothing$ for all $i$, and hence $\Gamma_{\infty}=\Sigma^{\prime}$.

Lemma 4.7. Let $\Gamma$ be a curve consisting of subcurves $\Gamma_{1}, \Gamma_{2}, \cdots$, and coinciding with $\Sigma^{*}$ on $\Gamma-\bigcap_{i=1}^{\infty} \Gamma_{i}$. Let $\Gamma_{i}, \Sigma_{i}^{*}$ and $\Omega_{*}^{*}$ be the corresponding connected components of $\Gamma-\left(\Gamma \cap \Sigma^{*}\right), \Sigma^{*}-\left(I \cap \Sigma^{*}\right)$ and $\Omega^{*}$. Then $g(\Gamma) \leqq \Sigma^{*}$ if $g\left(\Gamma_{i}\right) \leqq \Sigma_{i}^{*}$ for all $i=1,2, \cdots$.

Proof. (Figure 16)

$$
\begin{aligned}
\sum_{i=1}^{\infty} \Sigma_{i}^{*} \geqq \sum_{i=1}^{\infty} g\left(\Gamma_{i}\right) & =-\sum_{i=1}^{\infty} \Gamma_{i}+\frac{1}{R} \sum_{i=1}^{\infty} \Omega_{i} \\
& =-\Gamma+\frac{1}{R} \Omega+\left(\Gamma \cap \Sigma^{*}\right) .
\end{aligned}
$$

On the other hand, $\Sigma^{*}=\sum_{i=1}^{\infty} \Sigma_{\iota}^{*}+\left(\Gamma \cap \Sigma^{*}\right)$. This and (4.12) imply the lemma.

Lemma 4.8. Let $\Omega$ be as in Theorem 4.1, $\Gamma$ a circular are of radius $r<R$, then $g(\Gamma)<g\left(\Sigma^{\prime}\right)$ still holds.

Proof. Let $P$ and $Q$ be the end points of $\Gamma$ on $\Sigma$.

(i) Suppose we can draw in $\Omega$ a smaller circular arc $\Gamma^{\top}(R)$ of radius $R$ with $P$ and $Q$ as its end points (Figure 17a). Then by Lemma 2.2 and Lemma 2.3 we have

$$
g(\Gamma)<g(\Gamma(R))<g\left(\Sigma^{\prime}\right) .
$$

(ii) Suppose $\Gamma(R)$ does not lie inside $\Omega$. Then we can "shrink $\Gamma$ toward $\Sigma^{*}$ " until the first point of contact happens, i.e., consider all circular ares joining $P$ and $Q$, which lie between $\Gamma$ and $\Sigma^{*}$. Let $\Gamma\left(t_{1}\right)$ be the circular arc which has the first point of contact with $\Sigma^{*}$, at $P_{1}$ (Figure $17 \mathrm{~b}$ ). Then by the remarks of Lemma 2.2 we have

$$
g(\Gamma)<g\left(\Gamma^{\prime}\left(t_{1}\right)\right) .
$$

If Case (i) holds on each piece of $T_{P P_{1}}(t)$ and $\Gamma_{P_{1} Q}(t)$, then by Lemma 4.7, we will have

$$
g(\Gamma)<g\left(\Sigma^{\prime}\right)
$$


If Case (i) does hold on both of $\Gamma_{P P_{1}}\left(t_{1}\right)$ and $\Gamma_{P_{1} Q}\left(t_{1}\right)$, say it fails on $\Gamma_{P P_{1}}(t)$; then repeat the argument on $\Gamma_{P P_{1}}(t)$, we get a second point of contact and increase $g$ on the new circular arc. Since $\Sigma^{*}$ is of finite arc length, after at most countable process, we will get a sequence of circular $\operatorname{arcs} \Gamma_{1}, \Gamma_{2}, \cdots$, with each $\Gamma_{i}$ satisfying the situation in (i). Let $\Gamma_{i}(R)$ and $\Sigma_{i}^{*}$ be the circular arc of radius $R$ and boundary curves respectively corresponding to $\Gamma_{i}$, then Case (i) and Lemma 4.7 imply the lemma.

Lemmas $4.4,4.5,4.6$ and 4.7 together imply the following lemma.

Lemma 4.9. Let $\Omega$ be as in Theorem 4.1. Then $g(\Gamma)$ always achieves its maximum on $\Sigma^{\prime}$. More precisely, if $\Gamma \neq \varnothing$ or $\Sigma^{\prime}$ (i.e., $\Omega^{*} \neq \varnothing$ or $\left.\Omega\right)$, then $g(\Gamma)<\Sigma^{*}$ strictly.

Proof of Theorem 4.1. By Lemma 4.9

$$
g(\Gamma) \leqq g\left(\Sigma^{\prime}\right)=\Sigma^{*}
$$

On the other hand,

$$
\begin{aligned}
f(\Gamma) & =\Gamma+\frac{1}{R} \Omega^{*} \\
& =\Sigma-\left(-\Gamma+\frac{1}{R} \Omega^{\prime}\right), \quad \Omega^{\prime}=\Omega-\Omega^{*} .
\end{aligned}
$$

Again by Lemma $4.9,-\Gamma+(1 / R) \Omega^{\prime}$ achieves its maximum on $\Sigma^{*}$, hence

$$
\begin{aligned}
f(\Gamma) & \geqq \Sigma-\left(-\Sigma^{*}+\frac{1}{R} \Omega^{\prime}\left(\Sigma^{*}\right)\right) \\
& =\Sigma-\left(-\Sigma^{*}+\frac{1}{R} \Omega\right) \\
& =\Sigma-\left(-\Sigma^{*}+\Sigma\right) \\
& =\Sigma^{*}
\end{aligned}
$$

(4.13) and (4.14) imply

$$
g(\Gamma) \leqq \Sigma^{*} \leqq f(\Gamma)
$$

for all possible $\Gamma$. If $\Omega^{*} \neq \varnothing$ or $\Omega$ (4.15) holds strictly and hence by Theorem (3.4), Equation 3.1 has a solution for all contact angle $\gamma$.

Lemma 4.10. Let $\Gamma$ be a circular arc of radius $R$ in $\Omega$ which contacts to $\Sigma$ at $P$ and $Q$ (Figure 18). Suppose that no points of $\Sigma^{\prime}$ 
can be contacted by a circle of radius $R=\Omega / \Sigma$ in the interior of $\Omega$. Then $g(\Gamma)>\Sigma^{*}$.

Proof. We will prove that $g$ increases strictly as $\Sigma^{\prime}$ shrinks to $\Gamma$, and hence $g(\Gamma)>g\left(\Sigma^{\prime}\right)=\Sigma^{*}$.

Let $C_{1}, C_{2}, \cdots, C_{n}$ be a sequence of circular arc of radius $R$ in $\Omega$ which meet $\Sigma^{\prime}$ at $P_{1}, Q_{1} ; P_{2}, Q_{2} ; \cdots ; P_{n}, Q_{n}$ respectively, and let $\Gamma_{i}=$ $\Sigma_{P P_{i}}+C_{i}+\Sigma_{Q_{i} Q}, \Delta_{i}$ be the region between $C_{i}$ and $C_{i+1}$ (write $C_{0}=\Sigma_{P_{1} Q_{1}}^{\prime}$ ). (Figure 18). Then

$$
\begin{aligned}
g\left(\Sigma^{\prime}\right)-g\left(\Gamma_{1}\right) & =-\Sigma_{P_{1} Q_{1}}^{\prime}+\frac{1}{R} \Delta_{0}-C_{1} \\
& =\varphi\left(C_{0}\right)-\varphi\left(C_{1}\right)<0
\end{aligned}
$$

(Lemma 2.3 with $\Lambda=C_{1}$ )

if $C_{1}$ is properly chosen (all we require here is that $\Sigma_{P_{1} Q_{1}}<$ bigger circular arc passing through $P_{1}$ and $Q_{1}$ ).

Similarly

$$
\begin{aligned}
g\left(\Gamma_{1}\right)-g\left(\Gamma_{2}\right)= & -\Sigma_{P_{1} P_{2}}-C_{1}-\Sigma_{Q_{1} Q_{2}}+\frac{1}{R} \Delta_{1}+C_{2} \\
= & \varphi\left(\Sigma_{P_{1} P_{2}}+C_{1}+\Sigma_{Q_{1} Q_{2}}\right)-\varphi\left(C_{2}\right)<0 \\
& \left(\text { Lemma 3.2 with } \Lambda=C_{2}\right)
\end{aligned}
$$

if $\Gamma_{2}$ is properly chosen.

Repeat the argument we have

$$
g\left(\Gamma_{i}\right)<g\left(\Gamma_{i+1}\right)
$$

provided $\Gamma_{i+1}$ is properly chosen. For these sequence of $\Gamma_{i}$, we then have

$$
g\left(\Sigma^{\prime}\right)<g\left(\Gamma_{1}\right)<\cdots<g\left(\Gamma_{n}\right)<g(\Gamma) .
$$

Proof of Theorem 4.2. We are not going to prove this theorem in detail since much of the proof is essentially that of Theorem 4.1. We give here a sketch of the proof.

Let $\Gamma \in \mathscr{F}$, i.e., $\Gamma$ is a circular arc or a curve composed of cir. cular arcs of equal radii which are tangent to $\Sigma$ at regular points of $\Sigma$, and $\Gamma$ coincides with $\Sigma$ on the rest parts of $\Gamma$. By Lemma 4.7 it suffices to consider $g$ on each circular arc.

Let $C$ be one of the circular arc and let $r$ be its radius.

(i) If $r>R$, then repeat the argument as in the proof of 
Lemma 4.6. We can increase $g$ by moving $C$ towards $\Sigma^{\prime}$. We may not, however, reach $\Sigma^{\prime}$ since we do not know whether $\Sigma^{\prime}$ satisfies the interior rolling disk property of radius. If it does, then we will reach $\Sigma^{\prime}$ and hence $g(C)<g\left(\Sigma^{\prime}\right)=\Sigma^{*}$. If it does not, then as we have seen from the proof of Lemm 4.6, we shall stop at a curve which is the trajectory from rotating a circle of radius $R$ on $\Sigma^{\prime}$, i.e., a curve consisting of circular ares of radius $R$, all of which contact to $\Sigma^{\prime}$ (tangent to $\Sigma^{\prime}$ on regular points) and coinciding with $\Sigma^{\prime}$ on the complement of those circular arcs. By the hypothesis and Lemma 4.7 again, we still have $g(C)<\Sigma^{*}$.

(ii) If $r \leqq R$, then by Lemma 4.9, we can always increase $g$ by moving $C$ toward $\Sigma^{*}$ until it consists of circular arcs of radius $R$ which contact $\Sigma^{*}$. Again by the hypothesis and Lemma 4.7, we have $g(C)<\Sigma^{*}$. This completes the proof.

5. Existence and nonexistence of solutions on neck domains and tail domains. In $\S 4$ we proved the existence of solutions of (3.1) for all $\gamma$ on domains having the interior rolling disk property of radius $R=\Omega / \Sigma$. We will show in this section that this condition is also necessary for domains with no tail of radius $R$ but not necessary for some neck domains.

Let $\Omega$ be a plane domain with piecewise smooth boundary $\Sigma$ and $\Omega_{0}$ be any subregion of $\Omega$ with the interior rolling disk property of radius $R$. Consider the set

$$
\begin{array}{r}
S\left(\Omega, \Omega_{0}\right)=\left\{\Omega_{t} \mid \Omega_{0} \subset \Omega_{t} \subset \Omega, \Omega_{t}\right. \text { has the interior rolling } \\
\text { disk property of radius } R\} .
\end{array}
$$

Partially order $S$ by set inclusion and by Zorn's lemma, there exists a maximal element $\Omega_{1}$ in $S\left(\Omega, \Omega_{0}\right)$. If $\Omega$ has interior rolling disk property of radius $R$, then $\Omega_{1}=\Omega$ for every choice of $\Omega_{0}$. If $\Omega$ does not have the property, then $\Omega_{1} \subset \Omega$ strictly, and different choices of $\Omega_{0}$ may result in different $\Omega_{1}$. More precisely, $\Sigma_{1}$ (the boundary of $\Omega_{1}$ ) consists of circular arcs of radius $R$ and coincides with some subarc of $\Sigma$ on which a circle of radius $R$ can be contacted (rotated) in the interior of $\Omega$.

DeFinition 5.1. A plane domain $\Omega$ is called a neck domain of radius $R$ if it contains more than one maximal subregion with the interior rolling disk property of radius $R$ (Figure 19).

DeFinition 5.2. A plane domain $\Omega$ is said to have a tail of radius $R$ if there exists a subarc $\Sigma_{P Q}$ of $\Sigma$, such that

(i) $P$ and $Q$ can be contacted simultaneously by a circle of 
radius $R$ in the interior of $\Omega$.

(ii) No points of $\Sigma_{P Q}$ other than $P$ and $Q$ can be contacted by a circle of radius $R$ in the interior of $\Omega$ (Figure 19). With these definitions, we can now state our theorems.

Lemma 5.1. For tail domains of radius $R=\Omega / \Sigma$, there exists an $\gamma_{0}>0$ such that (3.1) has no solution for any $\gamma$ with $0 \leqq \gamma<\gamma_{0}$.

Proof. Lemma 4.10.

Theorem 4.1 and Lemma 5.1 together imply the following theorem.

THEOREM 5.2. Let $\Omega$ be a piecewise smooth domain with no necks of radius $R=\Omega / \Sigma$, then (3.1) has solutions for all $\gamma \geqq 0$ if and only if $\Omega$ satisfies the interior rolling disk condition of radius $R$.

For neck domains the interior rolling disk property is not a necessary condition for existence. A counterexample can be constructed as follows:

EXAMPLE 5.3. Let $\Omega_{1}$ and $\Omega_{2}$ be two unit disks with nonempty intersection, let $\Omega=\Omega_{1} \cup \Omega_{2},\{P, Q\}=\Sigma_{1} \cap \Sigma_{2}$, and $2 \theta$ be the angle made by $P, Q$ with respect to the center of the disk (Figure 20). Then we have

$$
\begin{aligned}
& \Omega=2 \pi-2 \theta+\sin 2 \theta \\
& \Sigma=4(\pi-\theta) \\
& R=\frac{\Omega}{\Sigma}=\frac{1}{2}\left[1+\frac{\sin 2 \theta}{2(\pi-\theta)}\right] \geqq \frac{1}{2} .
\end{aligned}
$$

Thus if the aperture $\overline{P Q}$ is less than $1, \Omega$ will be a neck domain of radius $R$. However, as we will see from the following proof, the solution surface always exists.

Let $\Gamma$ be the circular arc of radius $R$ passing through the points $P$ and $Q$ and call the angle subtended by the arc $2 \varphi$ (Figure 20). Let $S(R)$ be the region enclosed by $\Gamma$ and $\overline{P Q}$.

We have:

$$
\begin{aligned}
S(R) & =\frac{R^{2}}{2}(2 \rho-\sin 2 \varphi) \\
\Gamma & =2 R \varphi \\
\Omega^{*} & =\frac{1}{2} \Omega+S(R) \\
\Sigma^{*} & =\frac{1}{2} \Sigma .
\end{aligned}
$$


Thus

$$
\begin{gathered}
\left|\frac{\Omega^{*}}{\Omega}-\frac{\Sigma^{*}}{\Sigma}\right|=\frac{S(R)}{\Omega}=\frac{\frac{R^{2}}{2}(2 \varphi-\sin 2 \varphi)}{2 \pi-2 \theta+\sin 2 \theta} \\
\frac{\Gamma}{\Sigma}=\frac{R \varphi}{2(\pi-\theta)}
\end{gathered}
$$

(5.1), (5.2) and (5.3) imply:

$$
\begin{aligned}
\frac{I}{\Sigma}-\left|\frac{\Omega^{*}}{\Omega}-\frac{\Sigma^{*}}{\Sigma}\right| & =\frac{R}{2}\left[\frac{\varphi}{\pi-\theta}-\frac{2 \varphi-\sin 2 \varphi}{4(\pi-\theta)}\right] \\
& =\frac{R}{2(\pi-\theta)}[2 \varphi+\sin 2 \varphi]>0
\end{aligned}
$$

hence by Theorem 4.2, the solution exists.

In the case that $\Omega$ is the union of two disks of different radii, it was shown by Finn and Giusti [11] that the solution of (3.1) will fail to exist if the aperture is small enough. The following theorem is a generalization of this result.

THEOREM 5.4. Let $\Omega_{1}, \Omega_{2}$ be two plane domains with one region of overlap such that (3.1) has solutions on each domain for all $\gamma \geqq 0$. Suppose that $R_{1} \neq R_{2}\left(R_{i}=\Omega_{i} / \Sigma_{i}\right)$. There exists a positive number $\gamma_{0}$ such that (3.1) fails to have solutions on $\Omega=\Omega_{1} \cup \Omega_{2}$ for all $0 \leqq \gamma<\gamma_{0}$ when the aperture is sufficiently small.

Proof. Let $P, Q$ be the points of intersection of $\Sigma_{1}$ and $\Sigma_{2}$, let $\Gamma$ be the line segment joining $P$ and $Q$, and let $\Sigma^{*}=\Sigma \cap \Sigma_{1}$ (Figure 21), then we have

$$
\begin{gathered}
R=\frac{\Omega}{\Sigma} \longrightarrow \frac{\Omega_{1}+\Omega_{2}}{\Sigma_{1}+\Sigma_{2}}, \\
\Sigma^{*} \longrightarrow \Sigma_{1}, \quad \text { and } \quad \Omega^{*} \longrightarrow \Omega_{1} \text { as } \Gamma \longrightarrow 0
\end{gathered}
$$

and therefore

$$
\begin{aligned}
\left|\Sigma^{*}-\frac{1}{R} \Omega^{*}\right| & \longrightarrow\left|\Sigma_{1}-\frac{\Sigma_{1}+\Sigma_{2}}{\Omega_{1}+\Omega_{2}} \cdot \Omega_{1}\right| \\
& =\left|\frac{\Sigma_{1} \Omega_{2}-\Sigma_{2} \Omega_{1}}{\Omega_{1}+\Omega_{2}}\right|=\frac{\Omega_{1} \Omega_{2}}{\Omega_{1}+\Omega_{2}}\left|\frac{1}{R_{1}}-\frac{1}{R_{2}}\right| \\
& =\text { positive constant. }
\end{aligned}
$$

Hence

$$
\left|\Sigma^{*}-\frac{1}{R} \Omega^{*}\right|>\Gamma \text { if } \Gamma \text { is small enough. }
$$


This shows that solutions cannot exist for all $\gamma \geqq 0$ and the proof is completed.

With the results of Theorem 5.4 and Example 5.3, it seems natural to ask the following question: Can we expect the existence of solutions if $R_{1}=R_{2}$ ? The answer is still negative. Before giving a counterexample, we need the following lemma.

LEMMA 5.5. Let $\Omega_{1}$ and $\Omega_{2}$ be as is Theorem 5.4 except that $R_{1}=R_{2}$ and let $\Omega=\Omega_{1} \cup \Omega_{2}$. If $R=\Omega / \Sigma$, then $R \geqq R_{1}$, equality holds only if $\Omega_{1} \cap \Omega_{2}=\varnothing$ or $\Omega_{1}=\Omega_{2}$.

Proof. Let $\Delta_{i}, i=1, \cdots, n$ be the components of $\Omega_{1} \cap \Omega_{2}$ such that each $\Delta_{i}$ is bounded by $a_{i} \subset \Sigma_{i}$ and $b_{i} \subset \Sigma_{2}$ (Figure 22). Then we have

$$
\begin{aligned}
& \Omega=\Omega_{1}+\Omega_{2}-\sum_{i=1}^{n} \Delta_{i} \\
& \Sigma=\Sigma_{1}+\Sigma_{2}-\sum_{i=1}^{n}\left(a_{i}+b_{i}\right)
\end{aligned}
$$

hence

$$
\begin{aligned}
R-R_{1} & =\frac{\Omega \Sigma_{1}-\Omega_{1} \Sigma}{\Sigma \Sigma_{1}} \\
& =\frac{1}{\Sigma \Sigma_{1}}\left[\Omega_{2} \Sigma_{1}-\Omega_{1} \Sigma_{2}+\sum_{i=1}^{n}\left(\left(a_{i}+b_{i}\right) \Omega_{1}-\Delta_{i} \Sigma_{1}\right)\right] .
\end{aligned}
$$

Since $R_{1}=R_{2}$, we have $\Omega_{2} \Sigma_{1}-\Omega_{1} \Sigma_{2}=0$. Thus

$$
\begin{aligned}
R-R_{1} & =\frac{\Omega_{1}}{\Omega} \sum_{i=1}^{n}\left(\frac{a_{i}+b_{i}}{\Sigma_{1}}-\frac{\Delta_{i}}{\Omega_{1}}\right) \\
& \geqq \frac{\Omega_{1}}{\Sigma} \sum_{i=1}^{n}\left(\frac{b_{i}}{\Sigma_{1}}-\left|\frac{a_{i}}{\Sigma_{1}}-\frac{\Delta_{i}}{\Omega_{1}}\right|\right) .
\end{aligned}
$$

By hypothesis, a solution exists in $\Omega_{1}$ for each $\gamma$, hence (1.5) holds with $\Sigma^{*}=a_{i}, \Omega^{*}=\Delta_{i}$ and $\Gamma=b_{i}$. Therefore we have

$$
\left|\frac{a_{i}}{\Sigma_{i}}-\frac{\Delta_{i}}{\Omega_{1}}\right| \leqq \frac{b_{i}}{\Sigma_{1}}
$$

and hence $R-R_{1} \geqq 0$.

If $\Omega_{1} \cap \Omega_{2} \neq \varnothing$ and $\Omega_{1} \neq \Omega_{2}$ then (5.4) holds strictly for some $i$ and hence $R-R_{1}>0$ strictly.

From Lemma 4.5 , it is not difficult to construct a counterexample to our equation. The idea is as follows: Suppose we can construct a noncircular domain $\Omega_{1}$ which satisfies the interior rolling disk condition of radius 1 but does not satisfy the condition for any radius 
$>1$. Let $\Omega_{2}$ be the disk of radius 2 . We then have $R_{1}=R_{2}=1$ and $R>1$, thus $\Omega=\Omega_{1} \cup \Omega_{2}$ would be a tail domain of radius $R$ if the locations of $\Omega_{1}$ and $\Omega_{2}$ are properly chosen. Hence, by Theorem 5.2 no solution exists for $\gamma=0$.

EXAMPle 5.6. Let $\Omega_{1}$ be the figure shown in (Figure 23), whose boundary consists of two circular arcs of radius 1 and $a>1$ and the two line segments tangent to the circular arcs. Let $2 \theta$ be the angle formed by the extension of these two line segments. We then have:

$$
\begin{aligned}
& \Sigma_{1}=\pi(a+1)+2(a-1)(\theta+\cot \theta) \\
& \Omega_{2}=\frac{1}{2} \pi\left(a^{2}+1\right)+\left(a^{2}-1\right)(\theta+\cot \theta) .
\end{aligned}
$$

We want to choose $a$ and $\theta$ so that $R_{1}=\Omega_{1} / \Sigma_{1}=1$, or equivalently, we want $a$ and $\theta$ to satisfy the following equation:

$$
f(\theta)=\theta+\cot \theta=\frac{\pi}{2} \frac{1+2 a-a^{2}}{\left(1-a^{2}\right)} .
$$

Picking $a=3 / 2$ in (5.5), equation becomes:

$$
\theta+\cot \theta=\frac{7 \pi}{2}
$$

Since $f(0)>7 \pi / 2, f(\pi / 4)<7 \pi / 2$, there exists $\theta \in(0, \pi / 4)$ satisfying the above relation, more precisely, $\theta$ lies between $5^{\circ}$ and $6^{\circ}$.

Now let $\Omega_{2}$ be the disk of radius 2 , and let $\Omega=\Omega_{1} \cup \Omega_{2}$ with $\Omega_{2}$ overlapping $\Omega_{1}$ over the circular arc of radius $a$ (Figure 24). Then $R>1$, and $\Omega$ is a tail domain of radius $R$. Hence no solution exists for $\gamma=0$.

6. Two applications. In this section we shall derive two simple applications of our results. The first one is a purely geometrical property of plane figures. There may be a direct proof, however it can be derived easily from Theorem 4.1. The proof here is originally from Finn's proof for Bernstein's problem for constant mean curvature $H \neq 0$. The second one is an extension of the result of Concus and Finn [1], who proved that the solution of the capillary surface equation exists near a wedge of angle $2 \alpha,(0<\alpha<\pi / 2)$ if and only if $\alpha+\gamma \geqq \pi / 2$. We extend this result to the case $\alpha>\pi$.

THEOREM 6.1. If $\Omega$ is a piecewise smooth domain which satisfies the interior rolling disk condition of radius $R=\Omega / \Sigma$, then $\Omega$ cannot contain a disk of radius $2 R$ unless $\Omega$ is itself a disk of radius $2 R$. 
Proof. By Theorem 4.1, the equation

$$
\begin{gathered}
\operatorname{div} T u=H \text { in } \Omega \\
T u \cdot \nu=1 \text { on } \Sigma
\end{gathered}
$$

has a solution, where $H$ is implicitly determined by (6.1) and (6.2) and is equal to $\Sigma / \Omega=1 / R$.

Suppose $\Omega \supset B_{2 R}$, the disk of radius $2 R$, and $\Omega \neq B_{2 R}$. Then $\partial B_{2 R} \cap \Omega$ has positive one dimensional measure. Integrating (6.1) over $B_{2 R}$ and applying the divergence theorem, we get

$$
\begin{aligned}
H \cdot B_{2 R} & =\int_{\partial B_{2 R} \cap \Sigma} T u \cdot \nu+\int_{\partial B_{2 R} \cap \Omega} T u \cdot \nu \\
& <\partial B_{2 R} \cap \Sigma+\partial B_{2 R} \cap \Omega \\
& =\partial B_{2 R} .
\end{aligned}
$$

Or

$$
4 \pi R<4 \pi R
$$

which is impossible.

Equality can hold in (6.3) only if $\partial B_{2 R} \cap \Omega$ has one dimensional measure zero. The hypothesis that $\Sigma$ is piecewise smooth then implies $\Omega=B_{2 R}$.

REMARK. It is natural to generalize this result to $n$ dimensions. Suppose an $n$-dimensional set $\Omega$ has the property that a ball of radius $R=\Omega / \Sigma$ can be rotated along every point of its boundary $\Sigma$ in the interior of $\Omega$, then $\Omega$ cannot contain a ball of radius $n R$, unless $\Omega$ is itself a ball of radius $n R$. In this case our proof is no longer valid, since we only proved Theorem 4.1 in the 2 dimensional case.

THEOREM 6.2. In a wedge of angle $2 \alpha>\pi$, the equation

$$
\begin{aligned}
& \operatorname{div} T u=H \quad \text { inside the wedge } \\
& T u \cdot \nu=\cos \gamma \quad \text { on the boundary }
\end{aligned}
$$

has solutions for all contact angles $\gamma$.

Proof. Let $O$ be the vertex of the wedge and let $A$ and $B$ be two points on the boundary such that $\overline{O A}=\overline{O B}=1$. Consider the region

$$
\begin{aligned}
\Omega= & \{(x, y) \mid(x, y) \text { lie inside the wedge and the distance from } \\
& (x, y) \text { to } O A \text { or } O B \text { is less than } a\}
\end{aligned}
$$

(Figure 25), then 


$$
\begin{aligned}
& \Sigma=4+4 \alpha a \\
& \Omega=4 a+4 \alpha a^{2}-\pi a^{2} \\
& R=\frac{\Omega}{\Sigma}=a-\frac{\pi a^{2}}{4+4 \alpha a}<a
\end{aligned}
$$

thus $\Omega$ satisfies the interior rolling disk condition of radius $R$, and hence by Theorem 4.1 solutions exists for all $\gamma \geqq 0$.

\section{REFERENCES}

0. W. Blaschke, Kreis und Kugel, Walter de Gruyter and Co., Berlin, 1956.

1. P. Concus and R. Finn, On the behavior of a capillary surface in a wedge, Proc. Nat. Acad. Sci., 63 (1969), 292-299.

2. - On a class of capillary surfaces, J. d'Analyse Math., 23 (1970), 65-70.

3. On capillary free surfaces in the absence of gravity, Acta Math., 132 (1974), 177-198.

4. R.F. De Mar, A simple approach to isoperimetric problem in the plane, Math. Magazine, 48 (1975), 1-12.

5. P. Do Carmo, Differential Geometry of Curves and Surfaces, Prentice-Hall, New Jersey, 1976.

6. R. Finn, Remarks relevant to minimal surfaces, and to surfaces of prescribed mean curvature, J. d'Analyse Math., (1965), 139-160.

7. —_, A note on capillary free surfaces, Acta Math., 132 (1974), 199-205.

8. Capillarity phenomena, Uspekhi Mat. Nauk, Vol. 29, 4 (178), (1974).

9. — Existence and non-existence of capillary surfaces, Manuscripta Math., Vol. 28, Fasc. 1-3 (1979), 1-11.

10. R. Finn and E. Giusti, On nonparametric surfaces of constant mean curvature, Ann. Scu. Norm. Sup., Pisa, Ser IV, Vol. IV, No. 1, (1977).

11. — Non-existence and existence of capillary surfaces, Manuscripta Math., Vol. 28, Fasc. 1-3 (1979), 13-20.

12. D. Gilbarg and N. S. Trudinger, Elliptic Partial Differential Equations of Second Order, Springer-Verlag, New York-Heidelberg, 1977.

13. E. Guisti, Boundary value problems for nonparametric surfaces of prescribed mean curvature, Ann. Scu. Norm. Sup., Pisa, Ser. IV, Vol. III, No. 3, (1976), 501-548.

14. - Minimal Surfaces and Functions of Bounded Variation, Notes on Pure Math., Australian National Univ., Canberra, 1977.

15. - On the equation of surfaces of prescribed mean curvature.-Existence and uniqueness without boundary conditions, to appear.

16. R. Osserman, A Survey of Minimal Surfaces, Van Nostrand Reinhold, New York, 1969.

17. The isoperimetric inequality, Bulletin of the American Math. Soc., Vol. 84, No. 6 (1978), 1182-1238.

18. J. Serrin, The Dirichlet problem for surfaces of constant mean curvature, Proc. London Math. Soc., (3) 21 (1970), 361-384.

Received September 26, 1979.

National Tatwan University

Taipei, Taiwan, China 


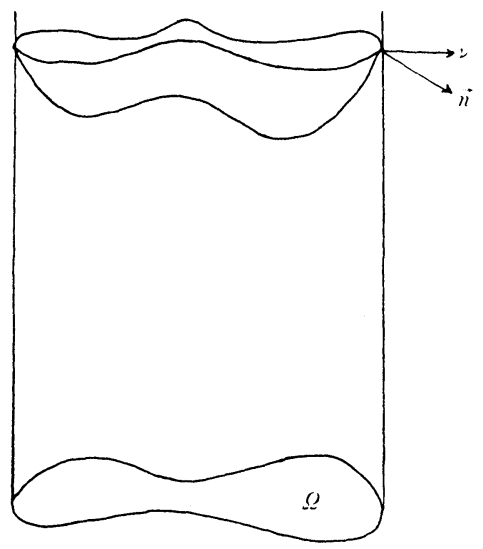

$$
\begin{aligned}
\vec{n} & =\left\langle\frac{u_{x}}{\sqrt{1+u_{x}^{2}+u_{y}^{2}}}, \frac{u_{y}}{\sqrt{1+u_{x}^{2}+u_{y}^{2}}}, \frac{-1}{\sqrt{1+u_{x}^{2}+u_{y}^{2}}}\right\rangle \\
& =\left\langle T u, \frac{-1}{\sqrt{1+u_{x}^{2}+u_{y}^{2}}}\right\rangle \\
\vec{n} \cdot \nu & =T u \cdot \nu=\cos \gamma
\end{aligned}
$$

FigURE 1

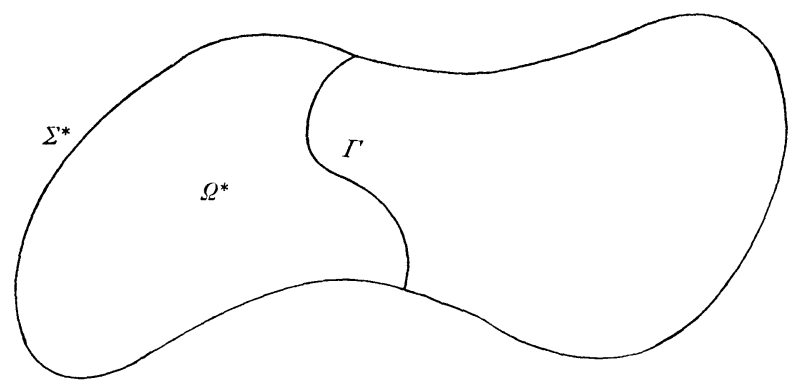

FIGURE 2 


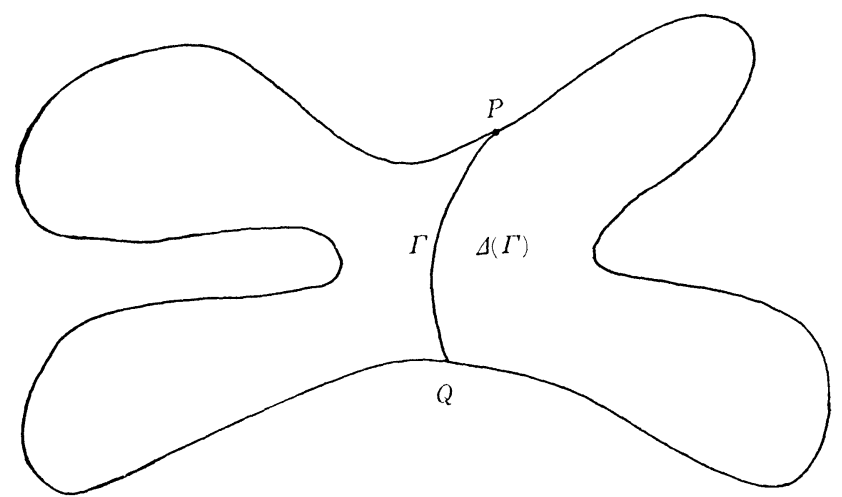

$\Gamma$ takes circular arc to bound the maximum area.

Figure $3 a$

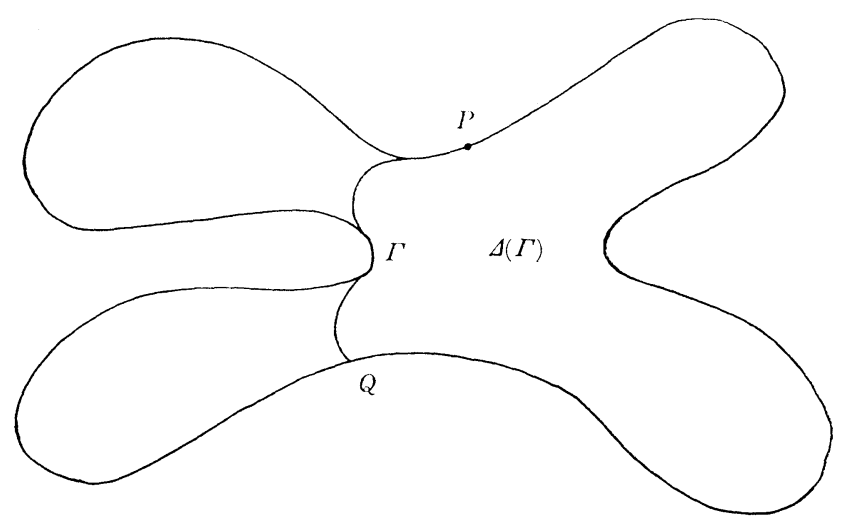

$\Gamma$ coincides with $\Sigma$ and circular arcs of equal radius, the circular arcs are tangent to $\Sigma$ at smooth points of $\Sigma$ except possibly for the two end points $P$ and $Q$.

Figure $3 \mathrm{~b}$

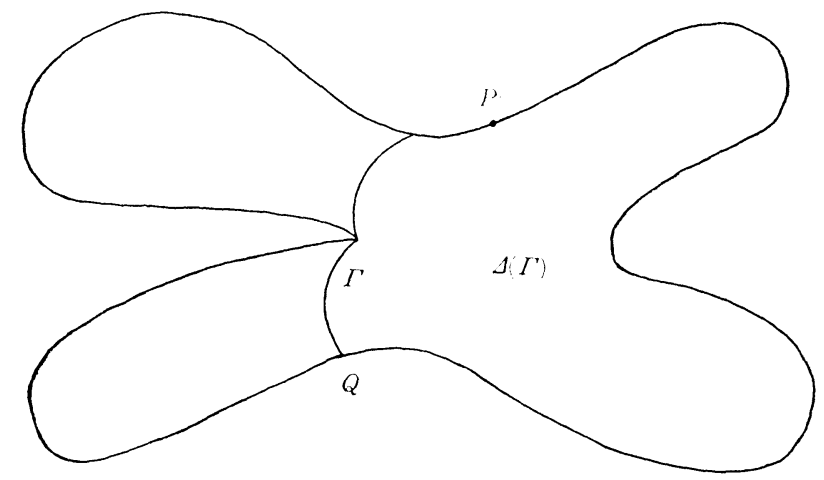

At singular points of $\Sigma, \Gamma$ may not be tangent to $\Sigma$.

FIGURE 3c 

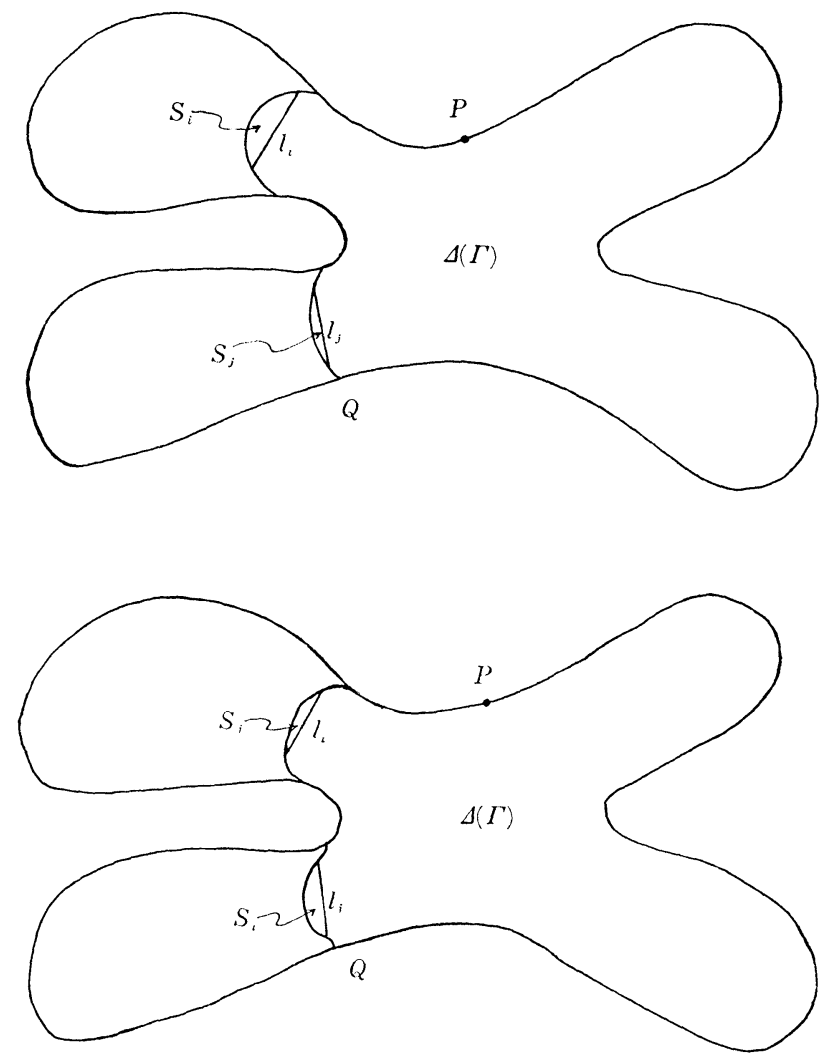

FigURE 4
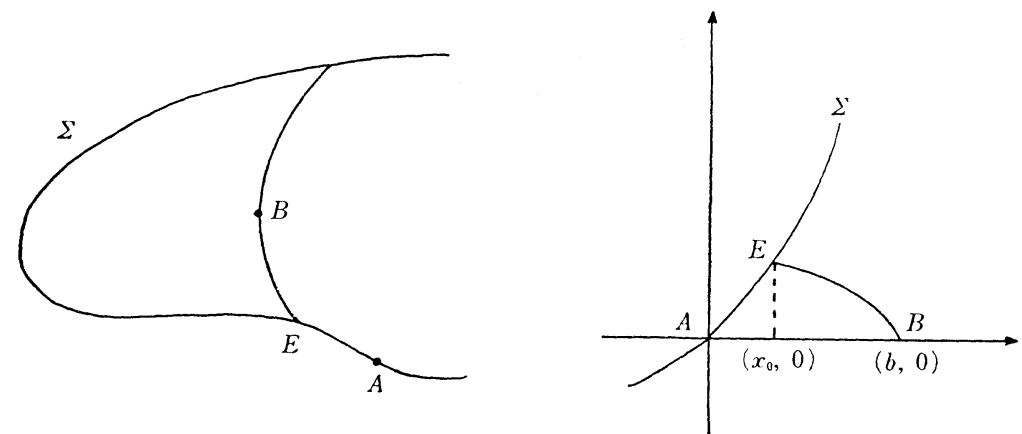

FigURE 5 


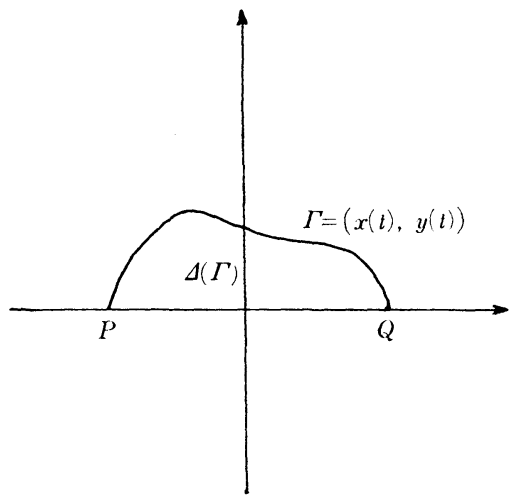

FigURE 6

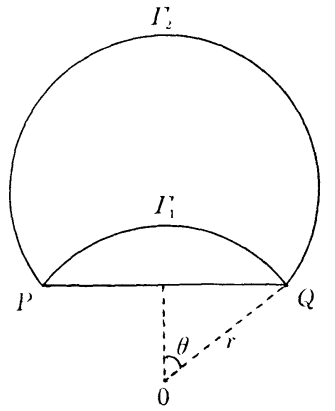

$\varphi$ achieves its local maximum on $\Gamma_{1}$.

Figure 7

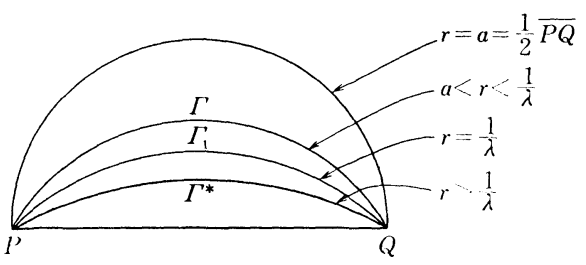

$$
\varphi(\Gamma)<\varphi\left(\Gamma_{1}\right), \varphi\left(\Gamma^{*}\right)<\varphi\left(\Gamma_{1}\right) .
$$

FIGURE 8

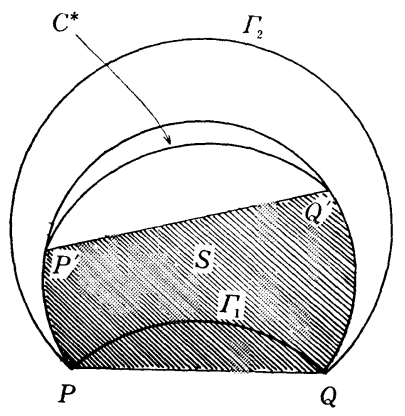

Figure 9 


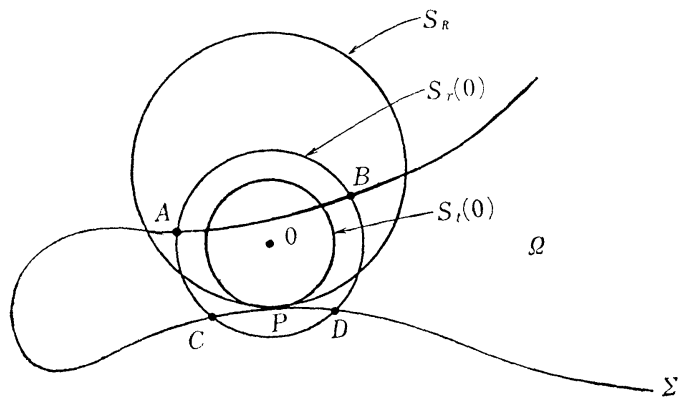

Figure 10

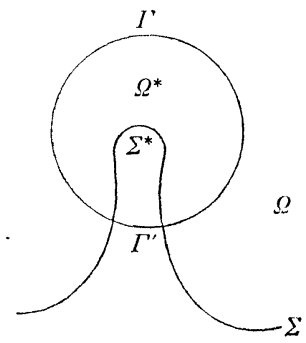

(a)

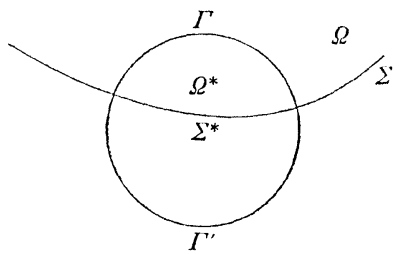

(b)

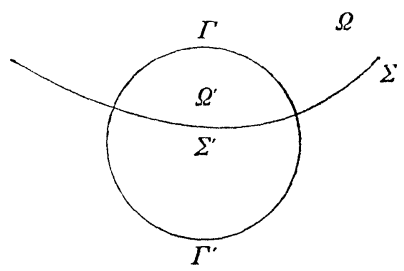

(c)

Figure 11 


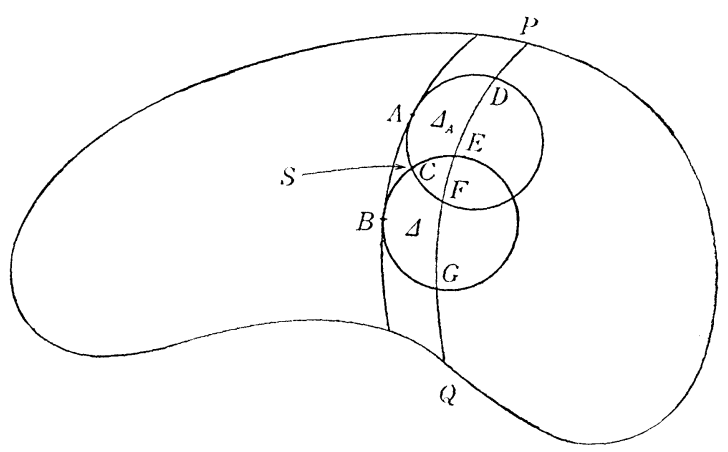

FIGURE 12

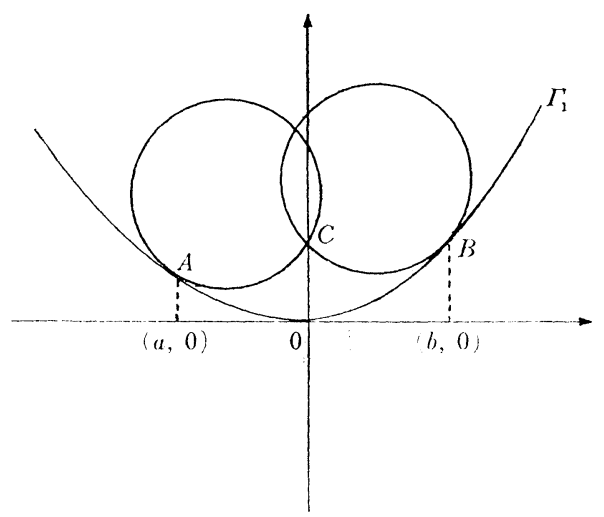

FigURE 13

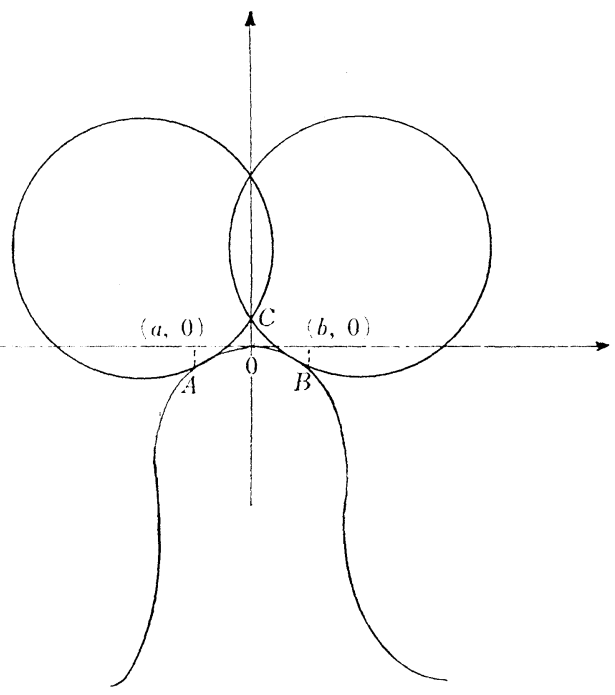

Figure 14 


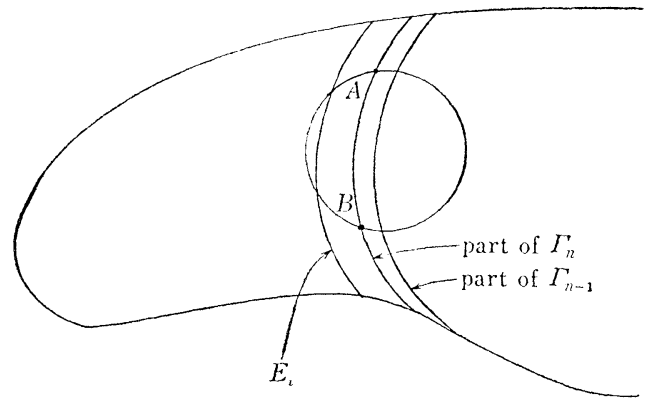

FigURE 15

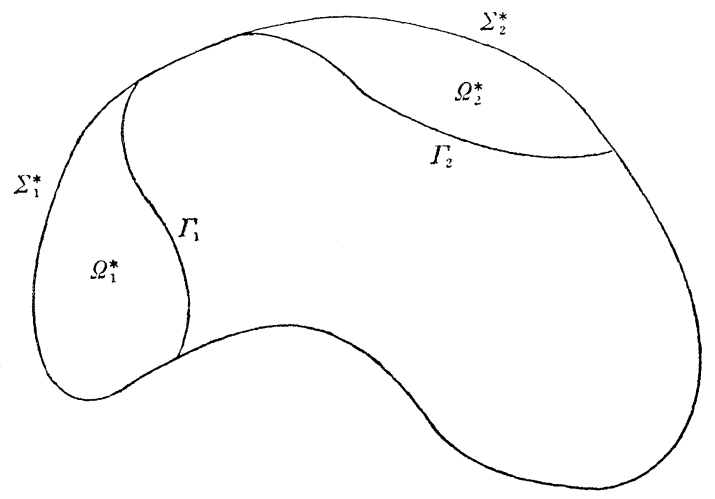

Figure 16

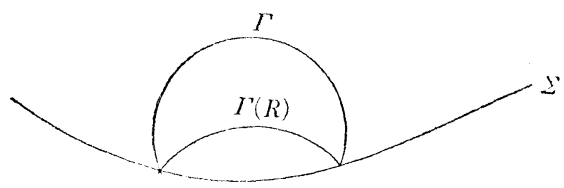

(a)

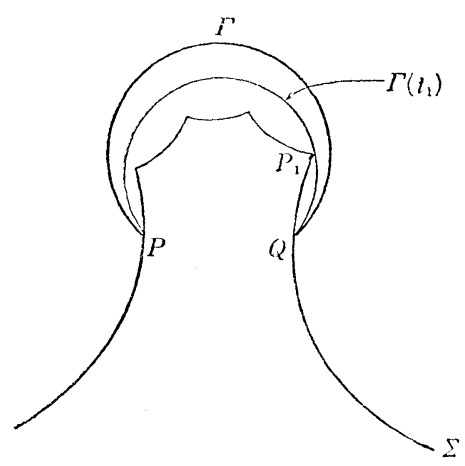

(b)

FigURE 17 


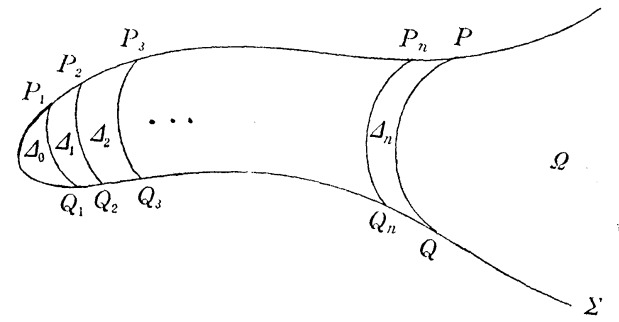

Figure 18

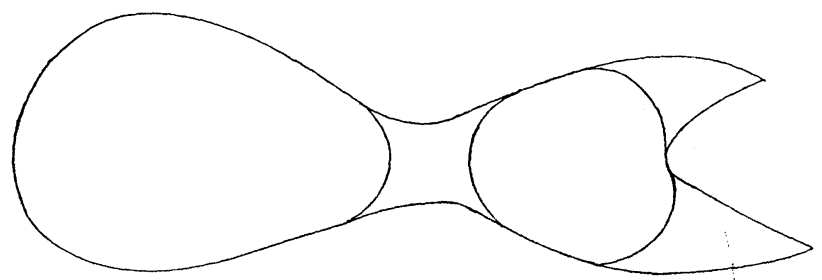

A neck domain with 2 tails.

Figure 19

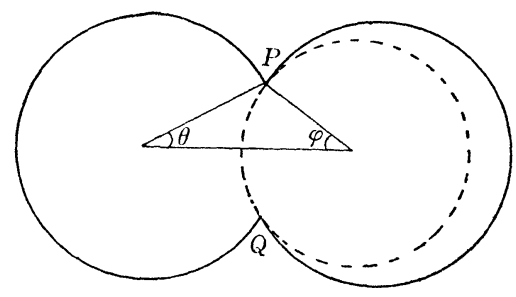

Figure 20

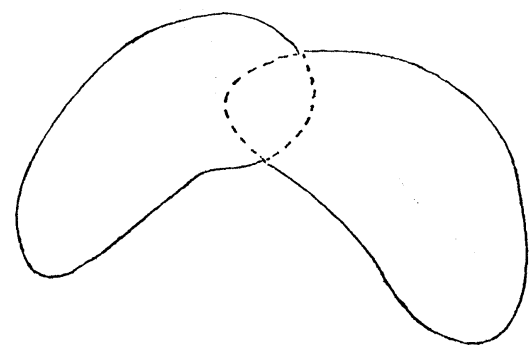

Figure 21 


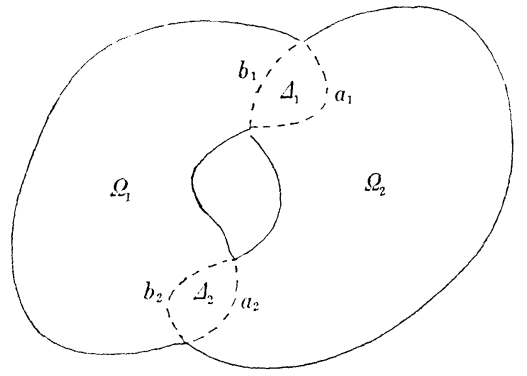

FIGURE 22

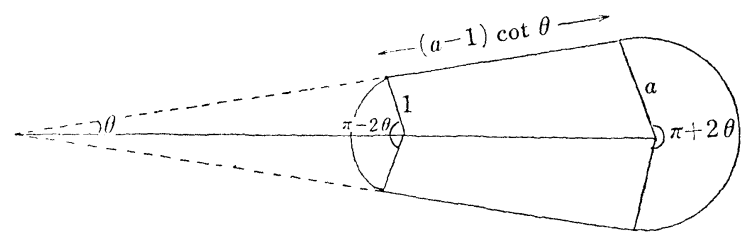

FigURe 23

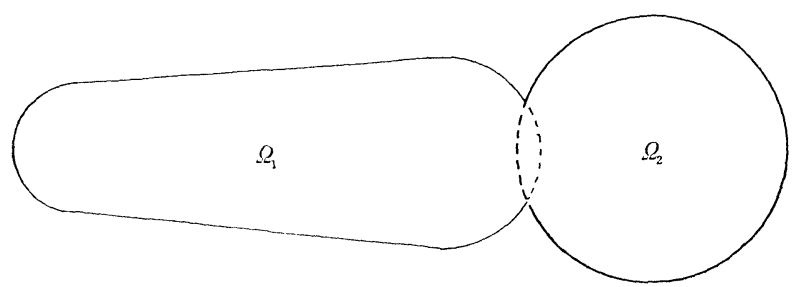

FIGURE 24

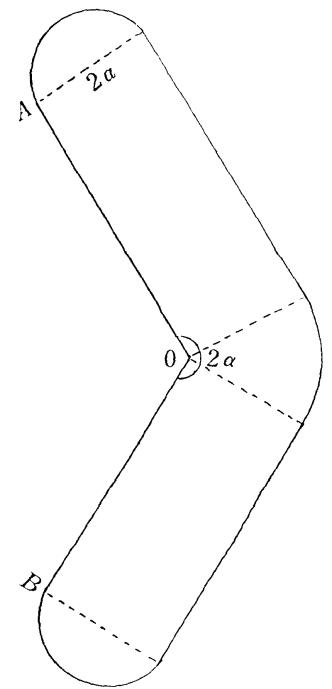

FIGURE 25 



\section{PACIFIC JOURNAL OF MATHEMATICS}

\section{EDITORS}

DONALD BABBITT (Managing Editor)

University of California

Los Angeles, CA 90024

Hugo Rossi

University of Utah

Salt Lake City, UT 84112

C. C. MOORE and ANDREW OGG

University of California

Berkeley, CA 94720
J. DUGUNDJI

Department of Mathematics

University of Southern California

Los Angeles, CA 90007

R. FinN and J. Milgram

Stanford University

Stanford, CA 94305

\section{ASSOCIATE EDITORS}
E. F. BECKENBACH
B. H. NeumanN
F. WOLF
K. YoSHIDA

\section{SUPPORTING INSTITUTIONS}

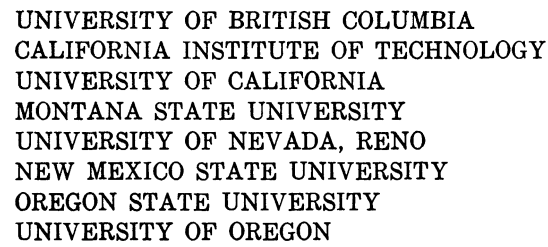

UNIVERSITY OF BRITISH COLUMBIA CALIFORNIA INSTITUTE OF TECHNOLOGY UNIVERSITY OF CALIFORNIA MONTANA STATE UNIVERSITY UNIVERSITY OF NEVADA, RENO NEW MEXICO STATE UNIVERSITY OREGON STATE UNIVERSITY UNIVERSITY OF OREGON

\author{
UNIVERSITY OF SOUTHERN CALIFORNIA \\ STANFORD UNIVERSITY \\ UNIVERSITY OF HAWAII \\ UNIVERSITY OF TOKYO \\ UNIVERSITY OF UTAH \\ WASHINGTON STATE UNIVERSITY \\ UNIVERSITY OF WASHINGTON
}

The Supporting Institutions listed above contribute to the cost of publication of this Journal, but they are not owners or publishers and have no responsibility for its content or policies.

Mathematical papers intended for publication in the Pacific Journal of Mathematics should be in typed form or offset-reproduced, (not dittoed), double spaced with large margins. Please do not use built up fractions in the text of the manuscript. However, you may use them in the displayed equations. Underline Greek letters in red, German in green, and script in blue. The first paragraph or two must be capable of being used separately as a synopsis of the entire paper. Please propose a heading for the odd numbered pages of less than 35 characters. Manuscripts, in triplicate, may be sent to any one of the editors. Please classify according to the scheme of Math. Reviews, Index to Vol. 39. Supply name and address of author to whom proofs should be sent. All other communications should be addressed to the managing editor, or Elaine Barth, University of California, Los Angeles, California, 90024.

50 reprints to each author are provided free for each article, only if page charges have been substantially paid. Additional copies may be obtained at cost in multiples of 50 .

The Pacific Journal of Mathematics is issued monthly as of January 1966. Regular subscription rate: $\$ 84.00$ a year (6 Vols., 12 issues). Special rato: $\$ 42.00$ a year to individual members of supporting institutions.

Subscriptions, orders for numbers issued in the last three calendar years, and changes of address shoud be sent to Pacific Journal of Mathematics, P.O. Box 969, Carmel Valley, CA 93924, U.S.A Old back numbers obtainable from Kraus Periodicals Co., Route 100, Millwood, NY 10546.

PUBLISHED BY PACIFIC JOURNAL OF MATHEMATICS, A NON-PROFIT CORPORATION

Printed at Kokusai Bunken Insatsusha (International Academic Printing Co., Ltd.). 8-8, 3-chome, Takadanobaba, Shinjuku-ku, Tokyo 160, Japan. 


\section{Pacific Journal of Mathematics \\ Vol. 88, No. $2 \quad$ April, 1980}

Reinhold Böhme, Stefan Hildebrandt and Engelbert Tausch, The two-dimensional analogue of the catenary ................. 247

Jean Ellen Taylor, Nonexistence of F-minimizing embedded disks ........ 279

Claus Gerhardt, A free boundary value problem for capillary surfaces ..... 285

Enrico Giusti, Generalized solutions for the mean curvature equation . . . . . 297

Jin-Tzu Chen, On the existence of capillary free surfaces in the absence of gravity.................................... 323

Leon M. Simon, Regularity of capillary surfaces over domains with corners.......................................... 363

Nicholas Jacob Korevaar, On the behavior of a capillary surface at a re-entrant corner.................................... 379

Henry Wente, The symmetry of sessile and pendent drops ............ 387

E. Gonzalez, Umberto Massari and I. Tamanini, Existence and regularity for the problem of a pendent liquid drop ...................... 399

Henry Wente, The stability of the axially symmetric pendent drop ........ 421

David Siegel, Height estimates for capillary surfaces . . . . . . . . . . . . 471

Bruce Edward Turkington, Height estimates for exterior problems of capillarity type ................................ 517

Robert Finn, The sessile liquid drop. I. Symmetric case .............. 541 